\title{
Dispersionless Hirota Equations of Two-Component BKP Hierarchy
}

\author{
Kanehisa TAKASAKI \\ Graduate School of Human and Environmental Studies, Kyoto University, \\ Yoshida, Sakyo, Kyoto 606-8501, Japan \\ E-mail: takasaki@math.h.kyoto-u.ac.jp
}

Received April 04, 2006, in final form May 02, 2006; Published online May 31, 2006

Original article is available at http://www.emis.de/journals/SIGMA/2006/Paper057/

\begin{abstract}
The BKP hierarchy has a two-component analogue (the 2-BKP hierarchy). Dispersionless limit of this multi-component hierarchy is considered on the level of the $\tau$-function. The so called dispersionless Hirota equations are obtained from the Hirota equations of the $\tau$-function. These dispersionless Hirota equations turn out to be equivalent to a system of Hamilton-Jacobi equations. Other relevant equations, in particular, dispersionless Lax equations, can be derived from these fundamental equations. For comparison, another approach based on auxiliary linear equations is also presented.
\end{abstract}

Key words: BKP hierarchy; Hirota equation; dispersionless limit

2000 Mathematics Subject Classification: 35Q58; 37K10; 58F07

\section{Introduction}

Among many variants of the KP hierarchy [1, 2], the BKP hierarchy [3, 4, 5, 6] enjoys a distinguished status. First of all, both the KP and BKP hierarchies are formulated by a single $\tau$-function [3]. The $\tau$-function of both hierarchies has a fermionic representation in terms of a one-component free fermion system [4]. The only difference lies in the type of fermions (charged or neutral). In the context of algebraic geometry, the KP hierarchy characterizes Jacobi varieties, and the BKP hierarchy corresponds to Prym varieties [5, 7, 8]. As regards the Lax formalism, both hierarchies are formulated by scalar pseudo-differential operators [6]. From this point of view, the BKP hierarchy is a reduction of the KP hierarchy. This relation carries over to the dispersionless limit [9].

The subject of this paper stems from the last topic, namely, the dispersionless BKP hierarchy. As for the general background on dispersionless limit of integrable hierarchies, we refer to Takasaki and Takebe's review [10].

Recently, Bogdanov and Konopelchenko studied the dispersionless BKP hierarchy from a new approach [11]. Their approach is based on the $\bar{\partial}$-dressing method [12, 13, 14, 15], which had been successfully applied to other dispersionless integrable hierarchies including the (genus zero) universal Whitham hierarchy [16]. Employing this method, they obtained an analogue of the so called "dispersionless Hirota equations" for the BKP hierarchy, which turns out to be slightly different from those of the KP and Toda hierarchies [10, 17, 18, 19, 20, 21].

The aim of this paper is to re-examine this issue along the lines of approach originally taken for the dispersionless Hirota equations $[10,17]$. Namely, we now start from the Hirota equations for the $\tau$-function itself rather than its dispersionless limit. Moreover, we consider the twocomponent BKP (2-BKP) hierarchy that includes two copies of the usual one-component BKP (1-BKP) hierarchy as a subset. 
Unlike the 1-BKP hierarchy, the 2-BKP hierarchy is not very well known in the literature (see Kac and van de Leur's paper [22] for a comprehensive account of this kind of multi-component integrable hierarchies). The 2-BKP hierarchy was first discovered as a system of Hirota equations [23]. A Lax representation was later proposed in an algebro-geometric framework [7, 8], which revealed that this system is closely related to two-dimensional finite-gap Schrödinger operators and their isospectral deformations (the Novikov-Veselov hierarchy) [24].

The 2-BKP hierarchy is rather special among multi-component integrable hierarchies, because it has a scalar Lax formalism as Shiota [7] and Krichever [8] presented. In this respect, the 2BKP hierarchy resembles the Toda hierarchy. The Toda hierarchy can be interpreted as the twocomponent KP (2-KP) hierarchy, though it has a scalar Lax formalism (in terms of difference operators) [25]. The scalar Lax formalism of the 2-BKP hierarchy, however, has a new feature. Namely, it has two spatial dimensions unlike the one-dimensional setting for the KP, Toda and 1-BKP hierarchies. This is the origin of all complexities that we shall encounter.

Our main concern lies in the structure of dispersionless Hirota equations and some other relevant equations in such a multi-component setting. We first derive a set of "differential Fay identities" from the Hirota equations (more precisely, their generating functional form). We then apply the standard prescription of dispersionless limit [10,17] (which is a kind of "quasi-classical limit") to these equations. The outcome is a set of equations that may be called dispersionless Hirota equations. Whereas part of them coincide with the equations presented by Bogdanov and Konopelchenko [11], other equations are seemingly new and specific to the two-component case. Moreover, we can correctly derive other relevant equations of the dispersionless hierarchy such as Hamilton-Jacobi equations, Lax equations, etc. We shall see that two distinct Poisson brackets show up in the formulation of dispersionless Lax equations. They are manifestation of presence of two spatial directions mentioned above.

Let us mention here that Konopelchenko and Moro have studied the dispersionless limit of the Novikov-Veselov hierarchy by means of $\bar{\partial}$-dressing [26]. Our results should be, in principle, related to theirs.

This paper is organized as follows. Sections 2 and 3 are devoted to a brief account of the tau function and equations satisfied by the tau functions. In Sections 4 and 5 , we derive the differential Fay identities and the dispersionless Hirota equations. In Sections 6 and 7, we examine their implications to the Hamilton-Jacobi equations and the dispersionless Lax equation. In Section 8, these results are compared with the conventional approach based on auxiliary linear equations.

\section{Tau functions and bilinear equations}

The 2-BKP hierarchy has two sets of time variables $t_{1}, t_{3}, \ldots, t_{2 n+1}, \ldots$ and $\bar{t}_{1}, \bar{t}_{3}, \ldots, \bar{t}_{2 n+1}, \ldots$ with odd indices ${ }^{1}$. For notational convenience, We collect these variables into two $\infty$-dimensional vectors

$$
\boldsymbol{t}=\left(t_{1}, t_{3}, \ldots\right), \quad \overline{\boldsymbol{t}}=\left(\bar{t}_{1}, \bar{t}_{3}, \ldots\right) .
$$

The $\tau$-functions $\tau=\tau(\boldsymbol{t}, \overline{\boldsymbol{t}})$ of this hierarchy are characterized by the fundamental bilinear equation $[23,22]$

$$
\begin{aligned}
& \oint \frac{d z}{2 \pi i z} e^{\xi\left(\boldsymbol{t}^{\prime}-\boldsymbol{t}, z\right)} \tau\left(\boldsymbol{t}^{\prime}-2\left[z^{-1}\right], \overline{\boldsymbol{t}}^{\prime}\right) \tau\left(\boldsymbol{t}+2\left[z^{-1}\right], \boldsymbol{t}\right) \\
& \quad=\oint \frac{d z}{2 \pi i z} e^{\xi\left(\overline{\boldsymbol{t}}^{\prime}-\overline{\boldsymbol{t}}, z\right)} \tau\left(\boldsymbol{t}^{\prime}, \overline{\boldsymbol{t}}^{\prime}-2\left[z^{-1}\right]\right) \tau\left(\boldsymbol{t}, \overline{\boldsymbol{t}}+2\left[z^{-1}\right]\right)
\end{aligned}
$$

\footnotetext{
${ }^{1}$ Throughout this paper, the bar " $\rightarrow$ " is used to create a new symbol, meaning nothing like complex conjugation. For instance, $t_{n}$ and $\bar{t}_{n}$ are understood to be independent variables.
} 
that holds for arbitrary values of $\boldsymbol{t}^{\prime}, \boldsymbol{t}, \overline{\boldsymbol{t}}^{\prime}$ and $\overline{\boldsymbol{t}}$. Both sides of this equation are understood to be contour integrals along the circle $|z|=R$ with sufficiently large radius $R$ and anti-clockwise orientation, i.e.,

$$
\oint \frac{d z}{2 \pi i z} f(z)=\oint_{|z|=R} \frac{d z}{2 \pi i z} f(z)
$$

Alternatively, these integrals may be thought of as a purely algebraic operation

$$
\oint \frac{d z}{2 \pi i z} \sum_{j \in \mathbb{Z}} a_{j} z^{j}=a_{0}
$$

for (formal or convergent) Laurent series. $\xi(\boldsymbol{t}, z)$ and $\left[z^{-1}\right]$ denote the BKP version

$$
\xi(\boldsymbol{t}, z)=\sum_{n=0}^{\infty} t_{2 n+1} z^{2 n+1}, \quad\left[z^{-1}\right]=\left(z^{-1}, \frac{z^{-3}}{3}, \ldots, \frac{z^{-2 n-1}}{2 n+1}, \ldots\right)
$$

of the usual notation for the KP hierarchy.

We can rewrite this bilinear equation to an infinite number of Hirota equations as follows. By substituting

$$
t_{n}^{\prime} \rightarrow t_{n}-x_{n}, \quad t_{n} \rightarrow t_{n}+x_{n}, \quad \bar{t}_{n}^{\prime} \rightarrow \bar{t}_{n}-\bar{x}_{n}, \quad \bar{t}_{n} \rightarrow \bar{t}_{n}+\bar{x}_{n},
$$

(2.1) transforms to

$$
\begin{aligned}
& \oint \frac{d z}{2 \pi i z} e^{2 \xi(\boldsymbol{x}, z)} \tau\left(\boldsymbol{t}-\boldsymbol{x}-2\left[z^{-1}\right], \overline{\boldsymbol{t}}-\overline{\boldsymbol{x}}\right) \tau\left(\boldsymbol{t}+\boldsymbol{x}+2\left[z^{-1}\right], \overline{\boldsymbol{t}}+\overline{\boldsymbol{x}}\right) \\
& =\oint \frac{d z}{2 \pi i z} e^{2 \xi(\overline{\boldsymbol{x}}, z)} \tau\left(\boldsymbol{t}-\boldsymbol{x}, \overline{\boldsymbol{t}}-\overline{\boldsymbol{x}}-2\left[z^{-1}\right]\right) \tau\left(\boldsymbol{t}+\boldsymbol{x}, \overline{\boldsymbol{t}}+\overline{\boldsymbol{x}}+2\left[z^{-1}\right]\right)
\end{aligned}
$$

where $\boldsymbol{x}=\left(x_{1}, x_{3}, \ldots\right)$ and $\overline{\boldsymbol{x}}=\left(\bar{x}_{1}, \bar{x}_{3}, \ldots\right)$. If we use the Hirota bilinear operators $D_{t_{2 n+1}}$ and $D_{\bar{t}_{2 n+1}}$ that act on the product of two functions $f(\boldsymbol{t}, \overline{\boldsymbol{t}}), g(\boldsymbol{t}, \overline{\boldsymbol{t}})$ as

$$
\begin{aligned}
& D_{t_{2 m+1}} f(\boldsymbol{t}, \overline{\boldsymbol{t}}) \cdot g(\boldsymbol{t}, \overline{\boldsymbol{t}})=\partial_{t_{2 n+1}} f(\boldsymbol{t}, \overline{\boldsymbol{t}}) \cdot g(\boldsymbol{t}, \overline{\boldsymbol{t}})-f(\boldsymbol{t}, \overline{\boldsymbol{t}}) \cdot \partial_{t_{2 n+1}} g(\boldsymbol{t}, \overline{\boldsymbol{t}}), \\
& D_{\bar{t}_{2 m+1}} f(\boldsymbol{t}, \overline{\boldsymbol{t}}) \cdot g(\boldsymbol{t}, \overline{\boldsymbol{t}})=\partial_{\bar{t}_{2 n+1}} f(\boldsymbol{t}, \overline{\boldsymbol{t}}) \cdot g(\boldsymbol{t}, \overline{\boldsymbol{t}})-f(\boldsymbol{t}, \overline{\boldsymbol{t}}) \cdot \partial_{\bar{t}_{2 n+1}} g(\boldsymbol{t}, \overline{\boldsymbol{t}}),
\end{aligned}
$$

the product of the $\tau$-functions in the integral can be expressed as

$$
\begin{aligned}
& \tau\left(\boldsymbol{t}+\boldsymbol{x}+2\left[z^{-1}\right], \overline{\boldsymbol{t}}+\overline{\boldsymbol{x}}\right) \tau\left(\boldsymbol{t}-\boldsymbol{x}-2\left[z^{-1}\right], \overline{\boldsymbol{t}}-\overline{\boldsymbol{x}}\right) \\
& =\exp \left(\xi\left(2 \tilde{\boldsymbol{D}}_{\boldsymbol{t}}, z\right)+\boldsymbol{x} \cdot \boldsymbol{D}_{\boldsymbol{t}}+\overline{\boldsymbol{x}} \cdot \boldsymbol{D}_{\overline{\boldsymbol{t}}}\right) \tau(\boldsymbol{t}, \overline{\boldsymbol{t}}) \cdot \tau(\boldsymbol{t}, \overline{\boldsymbol{t}}), \\
& \tau\left(\boldsymbol{t}+\boldsymbol{x}, \overline{\boldsymbol{t}}+\overline{\boldsymbol{x}}+2\left[z^{-1}\right]\right) \tau\left(\boldsymbol{t}-\boldsymbol{x}, \overline{\boldsymbol{t}}-\overline{\boldsymbol{x}}-2\left[z^{-1}\right]\right) \\
& =\exp \left(\xi\left(2 \tilde{\boldsymbol{D}}_{\overline{\boldsymbol{t}}}, z\right)+\boldsymbol{t} \cdot \boldsymbol{D}_{\boldsymbol{t}}+\overline{\boldsymbol{t}} \cdot \overline{\boldsymbol{D}}_{\overline{\boldsymbol{t}}}\right) \tau(\boldsymbol{t}, \overline{\boldsymbol{t}}) \cdot \tau(\boldsymbol{t}, \overline{\boldsymbol{t}}),
\end{aligned}
$$

where

$$
\begin{aligned}
& \tilde{\boldsymbol{D}}_{\boldsymbol{t}}=\left(D_{t_{1}}, \frac{1}{3} D_{t_{3}}, \ldots, \frac{1}{2 n+1} D_{t_{2 n+1}}, \ldots\right), \\
& \tilde{\boldsymbol{D}}_{\overline{\boldsymbol{t}}}=\left(D_{\bar{t}_{1}}, \frac{1}{3} D_{\bar{t}_{3}}, \ldots, \frac{1}{2 n+1} D_{\bar{t}_{2 n+1}}, \ldots\right), \\
& \boldsymbol{x} \cdot \boldsymbol{D}_{\boldsymbol{t}}=\sum_{n=0}^{\infty} x_{2 n+1} D_{t_{2 n+1}}, \quad \overline{\boldsymbol{x}} \cdot \boldsymbol{D}_{\overline{\boldsymbol{t}}}=\sum_{n=0}^{\infty} \bar{x}_{2 n+1} D_{\bar{t}_{2 n+1}} .
\end{aligned}
$$


Let us introduce the polynomials $q_{j}(\boldsymbol{t}), j=0,1,2, \ldots$ defined by the generating function

$$
e^{\xi(\boldsymbol{t}, z)}=\sum_{j=0}^{\infty} q_{j}(\boldsymbol{t}) z^{j} .
$$

We can thereby rewrite (2.1) as

$$
\begin{aligned}
& \sum_{j=0}^{\infty} q_{j}(2 \boldsymbol{x}) q_{j}\left(2 \tilde{\boldsymbol{D}}_{\boldsymbol{t}}\right) \exp \left(\boldsymbol{x} \cdot \boldsymbol{D}_{\boldsymbol{t}}+\overline{\boldsymbol{x}} \cdot \boldsymbol{D}_{\overline{\boldsymbol{t}}}\right) \tau(\boldsymbol{t}, \overline{\boldsymbol{t}}) \cdot \tau(\boldsymbol{t}, \overline{\boldsymbol{t}}) \\
& \quad=\sum_{j=0}^{\infty} q_{j}(2 \overline{\boldsymbol{x}}) q_{j}\left(2 \tilde{\boldsymbol{D}}_{\overline{\boldsymbol{t}}}\right) \exp \left(\boldsymbol{x} \cdot \boldsymbol{D}_{\boldsymbol{t}}+\overline{\boldsymbol{x}} \cdot \boldsymbol{D}_{\overline{\boldsymbol{t}}}\right) \tau(\boldsymbol{t}, \overline{\boldsymbol{t}}) \cdot \tau(\boldsymbol{t}, \overline{\boldsymbol{t}}) .
\end{aligned}
$$

Taylor expansion of this equation with respect to $\boldsymbol{x}$ and $\overline{\boldsymbol{x}}$ gives an infinite number of Hirota equations. In other words, this is a generating functional form of those Hirota equations.

The 2-BKP hierarchy contains two copies of the 1-BKP hierarchy in the $\boldsymbol{t}$ - and $\overline{\boldsymbol{t}}$-sectors. These subhierarchies show up by setting $\overline{\boldsymbol{t}}^{\prime}=\overline{\boldsymbol{t}}$ or $\boldsymbol{t}^{\prime}=\boldsymbol{t}$. If we set $\overline{\boldsymbol{t}}^{\prime}=\overline{\boldsymbol{t}},(2.1)$ reduces to

$$
\oint \frac{d z}{2 \pi i z} e^{\xi\left(\boldsymbol{t}^{\prime}-\boldsymbol{t}, z\right)} \tau\left(\boldsymbol{t}^{\prime}-2\left[z^{-1}\right], \overline{\boldsymbol{t}}\right) \tau\left(\boldsymbol{t}+2\left[z^{-1}\right], \overline{\boldsymbol{t}}\right)=\tau\left(\boldsymbol{t}^{\prime}, \overline{\boldsymbol{t}}\right) \tau(\boldsymbol{t}, \overline{\boldsymbol{t}}) .
$$

Similarly, setting $\boldsymbol{t}^{\prime}=\boldsymbol{t}$ yields

$$
\oint \frac{d z}{2 \pi i z} e^{\xi\left(\overline{\boldsymbol{t}}^{\prime}-\overline{\boldsymbol{t}}, z\right)} \tau\left(\boldsymbol{t}, \overline{\boldsymbol{t}}^{\prime}-2\left[z^{-1}\right]\right) \tau\left(\boldsymbol{t}, \overline{\boldsymbol{t}}+2\left[z^{-1}\right]\right)=\tau\left(\boldsymbol{t}, \overline{\boldsymbol{t}}^{\prime}\right) \tau(\boldsymbol{t}, \overline{\boldsymbol{t}}) .
$$

These equations are exactly the bilinear equations of the 1-BKP hierarchy $[3,4]$.

\section{Fermionic representation of tau functions}

The $\tau$-functions of the 1-BKP and 2-BKP hierarchies have a fermionic representation in terms of neutral fermions $[4,22,23]$. Let $\varphi_{j}, j \in \mathbb{Z}$, denote the generators of a Clifford algebra with the anti-commutation relations

$$
\left[\varphi_{j}, \varphi_{k}\right]_{+}=(-1)^{j} \delta_{j+k, 0}
$$

and $|0\rangle$ and $\langle 0|$ the vacuum states of the Fock and dual Fock spaces that are annihilated by half of these generators ("annihilation operators") as

$$
\langle 0| \varphi_{j}=0 \quad \text { for } j>0, \quad \varphi_{j}|0\rangle=0 \quad \text { for } j<0 .
$$

This is the fundamental setting of the neutral fermion system. Vacuum states of this system are two-dimensional. Namely, there are another pair of states $|1\rangle$ and $\langle 1|$ that are annihilated by the same set of annihilation operators. These vacuum states are interchanged by the action of $\sqrt{2} \varphi_{0}$ as

$$
\sqrt{2} \varphi_{0}|0\rangle=|1\rangle, \quad \sqrt{2} \varphi_{0}|1\rangle=|0\rangle, \quad\langle 0| \sqrt{2} \varphi_{0}=\langle 1|, \quad\langle 1| \sqrt{2} \varphi_{0}=\langle 0| .
$$

Now suppose that $g$ is an invertible element of the Clifford algebra that generates a "Bogolioubov transformation" of the form

$$
g \varphi_{k} g^{-1}=\sum_{j \in \mathbb{Z}} a_{j k} \varphi_{j}
$$


on the linear space spanned by $\phi_{h}$ 's. A typical (generic) case is the exponential

$$
g=\exp \left(\frac{1}{2} \sum_{j, k \geq 0} a_{j k} \varphi_{j} \varphi_{k}\right)
$$

of a fermionic bilinear form. Such an operator determines a $\tau$-function of the 2-BKP hierarchy as the vacuum expectation value

$$
\tau(\boldsymbol{t}, \overline{\boldsymbol{t}})=\left\langle 0\left|e^{H(\boldsymbol{t})} g e^{-\bar{H}(\overline{\boldsymbol{t}})}\right| 0\right\rangle,
$$

where $H(\boldsymbol{t})$ and $\bar{H}(\overline{\boldsymbol{t}})$ are linear combinations

$$
H(\boldsymbol{t})=\sum_{n=0}^{\infty} t_{2 n+1} H_{2 n+1}, \quad \bar{H}(\overline{\boldsymbol{t}})=\sum_{n=0}^{\infty} \bar{t}_{2 n+1} H_{-2 n-1}
$$

of the "Hamiltonians"

$$
H_{2 n+1}=\frac{1}{2} \sum_{j \in \mathbb{Z}}(-1)^{j+1} \varphi_{j} \varphi_{-j-2 n-1} .
$$

Note that this resembles the fermionic representation of the $\tau$-function of the Toda hierarchy $[27,28]$.

The fundamental bilinear equation (2.1) is a consequence of the algebraic relation

$$
\sum_{j \in \mathbb{Z}}(-1)^{j} \varphi_{j} g \otimes \varphi_{-j} g=\sum_{j \in \mathbb{Z}}(-1)^{j} g \varphi_{j} \otimes g \varphi_{-j}
$$

of the foregoing operators and the "boson-fermion correspondence"

$$
\begin{aligned}
& \sqrt{2}\left\langle 1\left|e^{H(\boldsymbol{t})} \varphi(z)\right| V\right\rangle=X(\boldsymbol{t}, z)\left\langle 0\left|e^{H(\boldsymbol{t})}\right| V\right\rangle, \\
& \sqrt{2}\left\langle W\left|\varphi\left(z^{-1}\right) e^{-\bar{H}(\overline{\boldsymbol{t}})}\right| 1\right\rangle=X(\overline{\boldsymbol{t}}, z)\left\langle W\left|e^{-\bar{H}(\overline{\boldsymbol{t}})}\right| 0\right\rangle
\end{aligned}
$$

that holds for arbitrary states $\langle W|| V$,$\rangle in the Fock and dual Fock spaces. \varphi(z)$ and $X(\boldsymbol{t}, z)$ denote the free fermion field

$$
\varphi(z)=\sum_{j \in \mathbb{Z}} \varphi_{j} z^{j}
$$

and the corresponding vertex operators

$$
X(\boldsymbol{t}, z)=e^{\xi(\boldsymbol{t}, z)} e^{-2 D(\boldsymbol{t}, z)}, \quad D(\boldsymbol{t}, z)=\sum_{n=0}^{\infty} \frac{z^{-2 n-1}}{2 n+1} \partial_{t_{2 n+1}} .
$$

The operator $D(\boldsymbol{t}, z)$ plays a fundamental role in the formulation of dispersionless Hirota equations.

\section{Differential Fay identities}

Employing the method developed for the KP hierarchy [10, 17], we can derive four types of "differential Fay identities". They are derived from (2.1) by the following procedure.

1) Differentiate $(2.1)$ by $t_{1}^{\prime}$ and set

$$
\boldsymbol{t}^{\prime}=\boldsymbol{t}+2\left[\lambda^{-1}\right]+2\left[\mu^{-1}\right], \quad \overline{\boldsymbol{t}}^{\prime}=\overline{\boldsymbol{t}}
$$


2) Differentiate (2.1) by $\bar{t}_{1}^{\prime}$ and set

$$
\boldsymbol{t}^{\prime}=\boldsymbol{t}, \quad \overline{\boldsymbol{t}}^{\prime}=\overline{\boldsymbol{t}}+2\left[\lambda^{-1}\right]+2\left[\mu^{-1}\right] .
$$

3) Differentiate (2.1) by $t_{1}^{\prime}$ and set

$$
\boldsymbol{t}^{\prime}=\boldsymbol{t}+2\left[\lambda^{-1}\right], \quad \overline{\boldsymbol{t}}^{\prime}=\overline{\boldsymbol{t}}+2\left[\mu^{-1}\right] .
$$

4) Differentiate (2.1) by $\bar{t}_{1}^{\prime}$, and set

$$
\boldsymbol{t}^{\prime}=\boldsymbol{t}+2\left[\lambda^{-1}\right], \quad \overline{\boldsymbol{t}}^{\prime}=\overline{\boldsymbol{t}}+2\left[\mu^{-1}\right] .
$$

$\lambda$ and $\mu$ are arbitrary complex constants that sit on the far side of the integral contour, namely, $|\lambda|>R$ and $|\mu|>R$.

1) In this case, we obtain a bilinear equation of the form

$$
\begin{aligned}
& \oint \frac{d z}{2 \pi i z} \frac{(z+\lambda)(z+\mu)}{(z-\lambda)(z-\mu)}\left(z \tau\left(\boldsymbol{t}+2\left[\lambda^{-1}\right]+2\left[\mu^{-1}\right]-2\left[z^{-1}\right], \overline{\boldsymbol{t}}\right)\right. \\
& \left.\quad+\left(\partial_{t_{1}} \tau\right)\left(\boldsymbol{t}+2\left[\lambda^{-1}\right]+2\left[\mu^{-1}\right]-2\left[z^{-1}\right], \overline{\boldsymbol{t}}\right)\right) \tau\left(\boldsymbol{t}+2\left[z^{-1}\right], \overline{\boldsymbol{t}}\right) \\
& \quad=\oint \frac{d z}{2 \pi i z}\left(\partial_{t_{1}} \tau\right)\left(\boldsymbol{t}+2\left[\lambda^{-1}\right]+2\left[\mu^{-1}\right], \overline{\boldsymbol{t}}-2\left[z^{-1}\right]\right) \tau\left(\boldsymbol{t}, \overline{\boldsymbol{t}}+2\left[z^{-1}\right]\right),
\end{aligned}
$$

where $\left(\partial_{t_{1}} \tau\right)(\boldsymbol{t}, \overline{\boldsymbol{t}})$ denotes the $t_{1}$-derivative of $\tau(\boldsymbol{t}, \overline{\boldsymbol{t}})$. Also the well known formula

$$
\sum_{n=0}^{\infty} \frac{2 z^{2 n+1}}{2 n+1}=\log \frac{1+z}{1-z}
$$

has been used to rewrite the exponential factor on the left hand side as

$$
e^{\xi\left(\boldsymbol{t}^{\prime}-\boldsymbol{t}, z\right)}=e^{\xi\left(2\left[\lambda^{-1}\right]+2\left[\mu^{-1}\right], z\right)}=\frac{(z+\lambda)(z+\mu)}{(z-\lambda)(z+\mu)} .
$$

Since the integrands on both sides of this bilinear equation are meromorphic functions on the far side $|z|>R$ of the integration contour, the integrals become the sums of their residues at poles. Whereas poles of the integrand on the left hand side are located at $z=\lambda, \mu, \infty$, the integrand on the right hand side has the only pole at $z=\infty$. We thus obtain a somewhat messy bilinear equation for special values of the $\tau$-function and its $t_{1}$-derivative. After some algebra, this bilinear equation boils down to the following relatively simple form that resembles the differential Fay identity of the KP hierarchy [10, 17]:

$$
\begin{aligned}
& \frac{\lambda+\mu}{\lambda-\mu}\left(\lambda-\mu-\partial_{t_{1}} \log \frac{\tau\left(\boldsymbol{t}+2\left[\lambda^{-1}\right], \overline{\boldsymbol{t}}\right)}{\tau\left(\boldsymbol{t}+2\left[\mu^{-1}\right], \overline{\boldsymbol{t}}\right)}\right) \\
& \quad=\left(\lambda+\mu-\partial_{t_{1}} \log \frac{\tau\left(\boldsymbol{t}+2\left[\lambda^{-1}\right]+2\left[\mu^{-1}\right], \overline{\boldsymbol{t}}\right)}{\tau(\boldsymbol{t}, \overline{\boldsymbol{t}})}\right) \frac{\tau\left(\boldsymbol{t}+2\left[\lambda^{-1}\right]+2\left[\mu^{-1}\right], \boldsymbol{t}\right) \tau(\boldsymbol{t}, \overline{\boldsymbol{t}})}{\tau\left(\boldsymbol{t}+2\left[\lambda^{-1}\right], \overline{\boldsymbol{t}}\right) \tau\left(\boldsymbol{t}+2\left[\mu^{-1}\right], \overline{\boldsymbol{t}}\right)} .
\end{aligned}
$$

Since these calculations are related to the $\boldsymbol{t}$-sector only, the same equation holds for the tau function of the 1-BKP hierarchy as well.

2) This case is essentially the same as case 1) except that the roles of $\boldsymbol{t}$ and $\overline{\boldsymbol{t}}$ are interchanged. The final form of the bilinear equation reads

$$
\frac{\lambda+\mu}{\lambda-\mu}\left(\lambda-\mu-\partial_{\bar{t}_{1}} \log \frac{\tau\left(\boldsymbol{t}, \overline{\boldsymbol{t}}+2\left[\lambda^{-1}\right]\right)}{\tau\left(\boldsymbol{t}, \overline{\boldsymbol{t}}+2\left[\mu^{-1}\right]\right)}\right)
$$




$$
=\left(\lambda+\mu-\partial_{\bar{t}_{1}} \log \frac{\tau\left(\boldsymbol{t}, \overline{\boldsymbol{t}}+2\left[\lambda^{-1}\right]+2\left[\mu^{-1}\right]\right)}{\tau(\boldsymbol{t}, \overline{\boldsymbol{t}})}\right) \frac{\tau\left(\boldsymbol{t}, \overline{\boldsymbol{t}}+2\left[\lambda^{-1}\right]+2\left[\mu^{-1}\right]\right) \tau(\boldsymbol{t}, \overline{\boldsymbol{t}})}{\tau\left(\boldsymbol{t}, \overline{\boldsymbol{t}}+2\left[\lambda^{-1}\right]\right) \tau\left(\boldsymbol{t}, \overline{\boldsymbol{t}}+2\left[\mu^{-1}\right]\right)} .
$$

3) In this case, we have a bilinear equation of the form

$$
\begin{aligned}
\oint \frac{d z}{2 \pi i z} & \frac{z+\lambda}{z-\lambda}\left(z \tau\left(\boldsymbol{t}+2\left[\lambda^{-1}\right]-2\left[z^{-1}\right], \overline{\boldsymbol{t}}+2\left[\mu^{-1}\right]\right)\right. \\
& \left.+\left(\partial_{t_{1}} \tau\right)\left(\boldsymbol{t}+2\left[\lambda^{-1}\right]-2\left[z^{-1}\right], \overline{\boldsymbol{t}}+2\left[\mu^{-1}\right]\right)\right) \tau\left(\boldsymbol{t}+2\left[z^{-1}\right], \overline{\boldsymbol{t}}\right) \\
& =\oint \frac{d z}{2 \pi i z} \frac{z+\mu}{z-\mu}\left(\partial_{t_{1}} \tau\right)\left(\boldsymbol{t}+2\left[\lambda^{-1}\right], \overline{\boldsymbol{t}}+2\left[\mu^{-1}\right]\right) \tau\left(\boldsymbol{t}, \overline{\boldsymbol{t}}+2\left[z^{-1}\right]\right) .
\end{aligned}
$$

Again by residue calculus, we obtain the following analogue of the differential Fay identity:

$$
\begin{aligned}
\lambda-\partial_{t_{1}} \log \frac{\tau\left(\boldsymbol{t}+2\left[\lambda^{-1}\right], \overline{\boldsymbol{t}}\right)}{\tau\left(\boldsymbol{t}, \overline{\boldsymbol{t}}+2\left[\mu^{-1}\right]\right)} & \\
& =\left(\lambda-\partial_{t_{1}} \log \frac{\tau\left(\boldsymbol{t}+2\left[\lambda^{-1}\right], \overline{\boldsymbol{t}}+2\left[\mu^{-1}\right]\right)}{\tau(\boldsymbol{t}, \overline{\boldsymbol{t}})}\right) \frac{\tau\left(\boldsymbol{t}+2\left[\lambda^{-1}\right], \overline{\boldsymbol{t}}+2\left[\mu^{-1}\right]\right) \tau(\boldsymbol{t}, \overline{\boldsymbol{t}})}{\tau\left(\boldsymbol{t}+2\left[\lambda^{-1}\right], \overline{\boldsymbol{t}}\right) \tau\left(\boldsymbol{t}+2\left[\mu^{-1}\right], \overline{\boldsymbol{t}}\right)} .
\end{aligned}
$$

Unlike the previous two equations, this equation has no counterpart in the 1-BKP hierarchy.

4) This case is parallel to case 3 ). The roles of $\boldsymbol{t}$ and $\overline{\boldsymbol{t}}$ are simply interchanged. The final form of the bilinear equation reads

$$
\begin{aligned}
\mu- & \partial_{\bar{t}_{1}} \log \frac{\tau\left(\boldsymbol{t}, \overline{\boldsymbol{t}}+2\left[\mu^{-1}\right]\right)}{\tau\left(\boldsymbol{t}+2\left[\lambda^{-1}\right], \overline{\boldsymbol{t}}\right)} \\
& =\left(\mu-\partial_{\bar{t}_{1}} \log \frac{\tau\left(\boldsymbol{t}+2\left[\lambda^{-1}\right], \overline{\boldsymbol{t}}+2\left[\mu^{-1}\right]\right)}{\tau(\boldsymbol{t}, \overline{\boldsymbol{t}})}\right) \frac{\tau\left(\boldsymbol{t}+2\left[\lambda^{-1}\right], \overline{\boldsymbol{t}}+2\left[\mu^{-1}\right]\right) \tau(\boldsymbol{t}, \overline{\boldsymbol{t}})}{\tau\left(\boldsymbol{t}+2\left[\lambda^{-1}\right], \overline{\boldsymbol{t}}\right) \tau\left(\boldsymbol{t}+2\left[\mu^{-1}\right], \overline{\boldsymbol{t}}\right)} .
\end{aligned}
$$

This equation, too, has no counterpart in the 1-BKP hierarchy.

We have thus derived the four types of equations, (4.1)-(4.4), as an analogue of the differential Fay identity of the KP hierarchy. The latter is known to be equivalent to the full KP hierarchy [10, Appendix B]. It seems likely that the same is also true for the 2-BKP hierarchy. Namely, we conjecture that the system of equations (4.1)-(4.4) will be equivalent to the 2-BKP hierarchy.

The subsequent consideration may be thought of as partial evidence that supports this conjecture. We shall examine the dispersionless limit of these equations, which will eventually turn out to be equivalent to the dispersionless limit of the 2-BKP hierarchy.

\section{Dispersionless Hirota equations}

As in the case of other integrable hierarchies [10], dispersionless limit is realized as a kind of "quasi-classical limit". To consider this limit in the language of $\tau$-function, we allow the $\tau$ function $\tau=\tau(\hbar, \boldsymbol{t}, \overline{\boldsymbol{t}})$ to depend on the "Planck constant" $\hbar$ and assume that the rescaled $\tau$-function

$$
\tau_{\hbar}(\boldsymbol{t}, \overline{\boldsymbol{t}})=\tau\left(\hbar, \hbar^{-1} \boldsymbol{t}, \hbar^{-1} \overline{\boldsymbol{t}}\right)
$$

behaves as

$$
\log \tau_{\hbar}(\boldsymbol{t}, \overline{\boldsymbol{t}})=\hbar^{-2} F(\boldsymbol{t}, \overline{\boldsymbol{t}})+O\left(\hbar^{-1}\right)
$$

in the classical limit $\hbar \rightarrow 0$. The goal of the following consideration is to derive a set of differential equations for the scaling function $F=F(\boldsymbol{t}, \overline{\boldsymbol{t}})$ from the differential Fay identities (4.1)-(4.4). 
Upon rescaling the time variables as above, the derivative operators and the bracket symbols in (4.1)-(4.4) are also rescaled as

$$
\partial_{t_{2 n+1}} \rightarrow \hbar \partial_{t_{2 n+1}}, \quad \partial_{\bar{t}_{2 n+1}} \rightarrow \hbar \partial_{\bar{t}_{2 n+1}}, \quad\left[\lambda^{-1}\right] \rightarrow \hbar\left[\lambda^{-1}\right], \quad\left[\mu^{-1}\right] \rightarrow \hbar\left[\mu^{-1}\right] .
$$

Bearing this in mind, let us examine the limit of these equations as $\hbar \rightarrow 0$.

1) Let us first consider (4.1). By rescaling, this equation transforms to

$$
\begin{aligned}
\frac{\lambda+\mu}{\lambda-\mu} & \left(\lambda-\mu-\hbar \partial_{t_{1}} \log \frac{\tau_{\hbar}\left(\boldsymbol{t}+2 \hbar\left[\lambda^{-1}\right], \overline{\boldsymbol{t}}\right)}{\tau_{\hbar}\left(\boldsymbol{t}+2 \hbar\left[\mu^{-1}\right], \overline{\boldsymbol{t}}\right)}\right) \\
= & \left(\lambda+\mu-\hbar \partial_{t_{1}} \log \frac{\tau_{\hbar}\left(\boldsymbol{t}+2 \hbar\left[\lambda^{-1}\right]+2 \hbar\left[\mu^{-1}\right], \overline{\boldsymbol{t}}\right)}{\tau_{\hbar}(\boldsymbol{t}, \overline{\boldsymbol{t}})}\right) \\
& \times \frac{\tau_{\hbar}\left(\boldsymbol{t}+2 \hbar\left[\lambda^{-1}\right]+2 \hbar\left[\mu^{-1}\right], \overline{\boldsymbol{t}}\right) \tau_{\hbar}(\boldsymbol{t}, \overline{\boldsymbol{t}})}{\tau_{\hbar}\left(\boldsymbol{t}+2 \hbar\left[\lambda^{-1}\right], \overline{\boldsymbol{t}}\right) \tau_{\hbar}\left(\boldsymbol{t}+2 \hbar\left[\mu^{-1}\right], \overline{\boldsymbol{t}}\right)} .
\end{aligned}
$$

This equation contains three types of "shifted" $\tau$-functions. We can use the already mentioned differential operator

$$
D(z)=D(\boldsymbol{t}, z)=\sum_{n=0}^{\infty} \frac{z^{-2 n-1}}{2 n+1} \partial_{t_{2 n+1}}
$$

to express these shifted $\tau$-functions as

$$
\begin{aligned}
& \tau_{\hbar}\left(\boldsymbol{t}+2 \hbar\left[\lambda^{-1}\right], \overline{\boldsymbol{t}}\right)=e^{2 \hbar D(\lambda)} \tau_{\hbar}(\boldsymbol{t}, \overline{\boldsymbol{t}}), \\
& \tau_{\hbar}\left(\boldsymbol{t}+2 \hbar\left[\mu^{-1}\right], \overline{\boldsymbol{t}}\right)=e^{2 \hbar D(\mu)} \tau_{\hbar}(\boldsymbol{t}, \overline{\boldsymbol{t}}), \\
& \tau_{\hbar}\left(\boldsymbol{t}+2 \hbar\left[\lambda^{-1}\right]+2 \hbar\left[\mu^{-1}\right], \overline{\boldsymbol{t}}\right)=e^{2 \hbar D(\lambda)+2 \hbar D(\mu)} \tau_{\hbar}(\boldsymbol{t}, \overline{\boldsymbol{t}}) .
\end{aligned}
$$

This enables us to evaluate the logarithm of " $\tau$-quotients" in the previous equation as

$$
\begin{aligned}
& \log \frac{\tau_{\hbar}\left(\boldsymbol{t}+2 \hbar\left[\lambda^{-1}\right], \overline{\boldsymbol{t}}\right)}{\tau_{\hbar}\left(\boldsymbol{t}+2 \hbar\left[\mu^{-1}\right], \overline{\boldsymbol{t}}\right)}=\left(e^{2 \hbar D(\lambda)}-e^{2 \hbar D(\mu)}\right) \log \tau_{\hbar}(\boldsymbol{t}, \overline{\boldsymbol{t}})=2 \hbar^{-1}(D(\lambda)-D(\mu)) F+O(1), \\
& \log \frac{\tau_{\hbar}\left(\boldsymbol{t}+2 \hbar\left[\lambda^{-1}\right]+2 \hbar\left[\mu^{-1}\right], \overline{\boldsymbol{t}}\right)}{\tau_{\hbar}(\boldsymbol{t}, \overline{\boldsymbol{t}})}=\left(e^{2 \hbar D(\lambda)+2 \hbar D(\mu)}-1\right) \log \tau_{\hbar}(\boldsymbol{t}, \overline{\boldsymbol{t}}) \\
& \quad=2 \hbar^{-1}(D(\lambda)+D(\mu)) F+O(1), \\
& \log \frac{\tau_{\hbar}\left(\boldsymbol{t}+2 \hbar\left[\lambda^{-1}\right]+2 \hbar\left[\mu^{-1}\right], \overline{\boldsymbol{t}}\right) \tau_{\hbar}(\boldsymbol{t}, \overline{\boldsymbol{t}})}{\tau_{\hbar}\left(\boldsymbol{t}+2 \hbar\left[\lambda^{-1}\right], \overline{\boldsymbol{t}}\right) \tau_{\hbar}\left(\boldsymbol{t}+2 \hbar\left[\mu^{-1}\right], \overline{\boldsymbol{t}}\right)} \\
& \quad=\left(e^{2 \hbar D(\lambda)+2 \hbar D(\mu)}+1-e^{2 \hbar D(\lambda)}-e^{2 \hbar D(\mu)}\right) \log \tau_{\hbar}(\boldsymbol{t}, \overline{\boldsymbol{t}})=4 D(\lambda) D(\mu) F+O(\hbar) .
\end{aligned}
$$

Having these results, we can readily find the limit of each terms in (5.2) as $\hbar \rightarrow 0$. This yields the equation

$$
\frac{\lambda+\mu}{\lambda-\mu}\left(\lambda-\mu-2 D(\lambda) \partial_{t_{1}} F+2 D(\mu) \partial_{t_{1}} F\right)=\left(\lambda+\mu-2 D(\lambda) \partial_{t_{1}} F-2 D(\mu) \partial_{t_{1}} F\right) e^{4 D(\lambda) D(\mu) F}
$$

for the $F$-function. We can rewrite this equation as

$$
\frac{p(\lambda)-p(\mu)}{p(\lambda)+p(\mu)}=\frac{\lambda-\mu}{\lambda+\mu} e^{4 D(\lambda) D(\mu) F},
$$

where $p(z)$ denotes the generating function

$$
p(z)=z-2 D(z) \partial_{t_{1}} F=z-\sum_{n=0}^{\infty} \frac{2 z^{-2 n-1}}{2 n+1} \partial_{t_{2 n+1}} \partial_{t_{1}} F
$$


of $\partial_{t_{2 n+1}} \partial_{t_{1}} F$. (5.3) is exactly the dispersionless Hirota equation derived by Bogdanov and Konopelchenko [11] for the 1-BKP hierarchy. We can also introduce the generating function

$$
S(z)=\sum_{n=0}^{\infty} t_{2 n+1} z^{2 n+1}-D(z) F
$$

of the first derivatives of $F$ to express $p(z)$ as

$$
p(z)=\partial_{t_{1}} S(z)
$$

and rewrite (5.3) as

$$
\frac{\partial_{t_{1}} S(\lambda)-\partial_{t_{1}} S(\mu)}{\partial_{t_{1}} S(\lambda)+\partial_{t_{1}} S(\mu)}=\frac{\lambda-\mu}{\lambda+\mu} e^{4 D(\lambda) D(\mu) F}
$$

2) We can start from (4.2) in the rescaled form

$$
\begin{aligned}
\frac{\lambda+\mu}{\lambda-\mu} & \left(\lambda-\mu-\hbar \partial_{\bar{t}_{1}} \log \frac{\tau_{\hbar}\left(\boldsymbol{t}, \overline{\boldsymbol{t}}+2 \hbar\left[\lambda^{-1}\right]\right)}{\tau_{\hbar}\left(\boldsymbol{t}, \overline{\boldsymbol{t}}+2 \hbar\left[\mu^{-1}\right]\right)}\right) \\
= & \left(\lambda+\mu-\hbar \partial_{\bar{t}_{1}} \log \frac{\tau_{\hbar}\left(\boldsymbol{t}, \overline{\boldsymbol{t}}+2 \hbar\left[\lambda^{-1}\right]+2 \hbar\left[\mu^{-1}\right]\right)}{\tau_{\hbar}(\boldsymbol{t}, \overline{\boldsymbol{t}})}\right) \\
& \times \frac{\tau_{\hbar}\left(\boldsymbol{t}, \overline{\boldsymbol{t}}+2 \hbar\left[\lambda^{-1}\right]+2 \hbar\left[\mu^{-1}\right]\right) \tau_{\hbar}(\boldsymbol{t}, \overline{\boldsymbol{t}})}{\tau_{\hbar}\left(\boldsymbol{t}, \overline{\boldsymbol{t}}+2 \hbar\left[\lambda^{-1}\right]\right) \tau_{\hbar}\left(\boldsymbol{t}, \overline{\boldsymbol{t}}+2 \hbar\left[\mu^{-1}\right]\right)}
\end{aligned}
$$

and repeat the same calculations as above. The outcome is the dispersionless Hirota equation

$$
\frac{\bar{p}(\lambda)-\bar{p}(\mu)}{\bar{p}(\lambda)+\bar{p}(\mu)}=\frac{\lambda-\mu}{\lambda+\mu} e^{4 \bar{D}(\lambda) \bar{D}(\mu) F}
$$

of the 1-BKP hierarchy in the $\overline{\boldsymbol{t}}$-sector. $\bar{D}(z)$ denotes the differential operator

$$
\bar{D}(z)=D(\overline{\boldsymbol{t}}, z)=\sum_{n=0}^{\infty} \frac{z^{-2 n-1}}{2 n+1} \partial_{\bar{t}_{2 n+1}}
$$

and $\bar{p}(z)$ the generating function

$$
\bar{p}(z)=z-2 \bar{D}(z) \partial_{\bar{t}_{1}} F=z-\sum_{n=0}^{\infty} \frac{2 z^{-2 n-1}}{2 n+1} \partial_{\bar{t}_{2 n+1}} \partial_{\bar{t}_{1}} F
$$

of $\partial_{\bar{t}_{2 n+1}} \partial_{\bar{t}_{1}} F$. We can also use the generating function

$$
\bar{S}(z)=\sum_{n=0}^{\infty} \bar{t}_{2 n+1} z^{2 n+1}-\bar{D}(z) F
$$

of the first derivatives of $F$ to express $\bar{p}(z)$ as

$$
\bar{p}(z)=\partial_{\bar{t}_{1}} \bar{S}(z)
$$

and to rewrite (5.4) as

$$
\frac{\partial_{\bar{t}_{1}} \bar{S}(\lambda)-\partial_{\bar{t}_{1}} \bar{S}(\mu)}{\partial_{\bar{t}_{1}} \bar{S}(\lambda)+\partial_{\bar{t}_{1}} \bar{S}(\mu)}=\frac{\lambda-\mu}{\lambda+\mu} e^{4 \bar{D}(\lambda) \bar{D}(\mu) F} .
$$


3) Let us now turn to (4.3). In the rescaled form, it reads

$$
\begin{aligned}
\lambda-\hbar \partial_{t_{1}} \log \frac{\tau_{\hbar}\left(\boldsymbol{t}+2 \hbar\left[\lambda^{-1}\right], \overline{\boldsymbol{t}}\right)}{\tau_{\hbar}\left(\boldsymbol{t}, \overline{\boldsymbol{t}}+2 \hbar\left[\mu^{-1}\right]\right)} \\
\quad=\left(\lambda-\hbar \partial_{t_{1}} \log \frac{\tau_{\hbar}\left(\boldsymbol{t}+2 \hbar\left[\lambda^{-1}\right], \overline{\boldsymbol{t}}+2 \hbar\left[\mu^{-1}\right]\right)}{\tau_{\hbar}(\boldsymbol{t}, \overline{\boldsymbol{t}})}\right) \frac{\tau_{\hbar}\left(\boldsymbol{t}+2 \hbar\left[\lambda^{-1}\right], \overline{\boldsymbol{t}}+2 \hbar\left[\mu^{-1}\right]\right) \tau_{\hbar}(\boldsymbol{t}, \overline{\boldsymbol{t}})}{\tau_{\hbar}\left(\boldsymbol{t}+2 \hbar\left[\lambda^{-1}\right], \overline{\boldsymbol{t}}\right) \tau_{\hbar}\left(\boldsymbol{t}+2 \hbar\left[\mu^{-1}\right], \overline{\boldsymbol{t}}\right)} .
\end{aligned}
$$

The logarithm of $\tau$-quotients in this equation can be evaluated in much the same way as the previous two cases. We thus obtain the equation

$$
\lambda-2 D(\lambda) \partial_{t_{1}} F+2 \bar{D}(\mu) \partial_{t_{1}} F=\left(\lambda-2 D(\lambda) \partial_{t_{1}} F-2 \bar{D}(\mu) \partial_{t_{1}} F\right) e^{4 D(\lambda) \bar{D}(\mu) F}
$$

for the $F$-function. In terms of the $S$-functions, this equation reads

$$
\frac{\partial_{t_{1}} S(\lambda)-\partial_{t_{1}} \bar{S}(\mu)}{\partial_{t_{1}} S(\lambda)+\partial_{t_{1}} \bar{S}(\mu)}=e^{4 D(\lambda) \bar{D}(\mu) F}
$$

Unlike the previous two equations, this equation has no counterpart in the 1-BKP hierarchy.

4) We can repeat the same calculations for (4.4) in the rescaled form

$$
\begin{aligned}
\mu- & \hbar \partial_{\bar{t}_{1}} \log \frac{\tau_{\hbar}\left(\boldsymbol{t}, \overline{\boldsymbol{t}}+2 \hbar\left[\mu^{-1}\right]\right)}{\tau_{\hbar}\left(\boldsymbol{t}+2 \hbar\left[\lambda^{-1}\right], \overline{\boldsymbol{t}}\right)} \\
& =\left(\mu-\hbar \partial_{\bar{t}_{1}} \log \frac{\tau_{\hbar}\left(\boldsymbol{t}+2 \hbar\left[\lambda^{-1}\right], \overline{\boldsymbol{t}}+2 \hbar\left[\mu^{-1}\right]\right)}{\tau_{\hbar}(\boldsymbol{t}, \overline{\boldsymbol{t}})}\right) \frac{\tau_{\hbar}\left(\boldsymbol{t}+2 \hbar\left[\lambda^{-1}\right], \overline{\boldsymbol{t}}+2 \hbar\left[\mu^{-1}\right]\right) \tau_{\hbar}(\boldsymbol{t}, \overline{\boldsymbol{t}})}{\tau_{\hbar}\left(\boldsymbol{t}+2 \hbar\left[\lambda^{-1}\right], \overline{\boldsymbol{t}}\right) \tau_{\hbar}\left(\boldsymbol{t}+2 \hbar\left[\mu^{-1}\right], \overline{\boldsymbol{t}}\right)}
\end{aligned}
$$

to obtain the equation

$$
\frac{\partial_{\bar{t}_{1}} \bar{S}(\mu)-\partial_{\bar{t}_{1}} S(\lambda)}{\partial_{\bar{t}_{1}} \bar{S}(\mu)+\partial_{\bar{t}_{1}} S(\lambda)}=e^{4 D(\lambda) \bar{D}(\mu) F}
$$

This equation, like (5.5), has no counterpart in the 1-BKP hierarchy.

As a final remark, let us note that the right hand sides of (5.5) and (5.6) coincide. This implies that the extra equation

$$
\frac{\partial_{t_{1}} S(\lambda)-\partial_{t_{1}} \bar{S}(\mu)}{\partial_{t_{1}} S(\lambda)+\partial_{t_{1}} \bar{S}(\mu)}=\frac{\partial_{\bar{t}_{1}} \bar{S}(\mu)-\partial_{\bar{t}_{1}} S(\lambda)}{\partial_{\bar{t}_{1}} \bar{S}(\mu)+\partial_{\bar{t}_{1}} S(\lambda)}
$$

or, equivalently,

$$
\partial_{t_{1}} S(\lambda) \cdot \partial_{\bar{t}_{1}} S(\lambda)=\partial_{t_{1}} \bar{S}(\mu) \cdot \partial_{\bar{t}_{1}} \bar{S}(\mu)
$$

holds. Since $\lambda$ and $\mu$ take arbitrary values, both sides of the last equation are actually independent of $\lambda$ and $\mu$. This value can be determined by letting $\lambda, \mu \rightarrow \infty$ :

$$
\lim _{\lambda \rightarrow \infty} \partial_{t_{1}} S(\lambda) \cdot \partial_{\bar{t}_{1}} S(\lambda)=\lim _{\mu \rightarrow \infty} \partial_{t_{1}} \bar{S}(\mu) \cdot \partial_{\bar{t}_{1}} \bar{S}(\mu)=-2 \partial_{t_{1}} \partial_{\bar{t}_{1}} F .
$$

Thus the extra equation reduces to

$$
\partial_{t_{1}} S(z) \cdot \partial_{\bar{t}_{1}} S(z)=\partial_{t_{1}} \bar{S}(z) \cdot \partial_{\bar{t}_{1}} \bar{S}(z)=u,
$$

where

$$
u=-2 \partial_{t_{1}} \partial_{\bar{t}_{1}} F
$$

As it will turn out later, this equation is the Hamilton-Jacobi equation of a two-dimensional Schrödinger equation that underlies the Novikov-Veselov hierarchy [24]. 


\section{Hamilton-Jacobi equations}

We show here that the dispersionless Hirota equations (5.3)-(5.6) are equivalent to a system of Hamilton-Jacobi equations for the $S$-functions. As we shall show later, these Hamilton-Jacobi equations are Lax equations of the dispersionless 2-BKP hierarchy in disguise. In other words, this system, too, is a special case of the universal Whitham hierarchy [16].

Following Teo's idea [21], we borrow the notion of "Faber polynomials" from the theory of univalent functions (see Teo's paper [21] and references cited therein). This enables us to do calculations in a clear and systematic way. The Faber polynomials are defined by a generating function, and eventually turn out to give the Hamiltonians of the Hamilton-Jacobi equations.

1) To derive Hamilton-Jacobi equations from (5.3), we first rewrite it to the logarithmic form

$$
\log \frac{p(\lambda)-p(\mu)}{p(\lambda)+p(\mu)}=\log \frac{\lambda-\mu}{\lambda+\mu}+\sum_{n, m=0}^{\infty} \frac{4 \lambda^{-2 n-1} \mu^{-2 m-1}}{(2 n+1)(2 m+1)} \partial_{t_{2 n+1}} \partial_{t_{2 m+1}} F .
$$

We now introduce the Faber polynomials $\Phi_{n}(p), n=1,2, \ldots$, of $p(z)$. They are defined by a generating function of the form

$$
\log \frac{p(z)-w}{z}=-\sum_{n=1}^{\infty} \frac{z^{-n}}{n} \Phi_{n}(w) .
$$

Although this definition is valid for any Laurent series $p(z)$ of the form $p(z)=z+O(1)$, the present setting is special in that $p(z)$ is an odd function:

$$
p(-z)=-p(z) \text {. }
$$

Because of this, we have the identity

$$
\log \frac{p(-z)+w}{-z}=\log \frac{p(z)-w}{z},
$$

which implies that $\Phi_{n}(w)$ is even if $n$ is even and odd if $n$ is odd, i.e.,

$$
\Phi_{n}(-w)=(-1)^{n} \Phi_{n}(w) .
$$

Therefore we have

$$
\log \frac{p(z)-w}{p(z)+w}=-\sum_{n=1}^{\infty} \frac{z^{-n}}{n}\left(\Phi_{n}(w)-\Phi_{n}(-w)\right)=-\sum_{n=0}^{\infty} \frac{2 z^{-2 n-1}}{2 n+1} \Phi_{2 n+1}(w) .
$$

Substituting $z=\lambda$ and $w=p(\mu)$ yields

$$
\log \frac{p(\lambda)-p(\mu)}{p(\lambda)+p(\mu)}=-\sum_{n=0}^{\infty} \frac{2 \lambda^{-2 n-1}}{2 n+1} \Phi_{2 n+1}(p(\mu)),
$$

which coincides with the left hand side of (6.1). As regards the right hand side of (6.1), we can rewrite the double sum as

$$
\begin{gathered}
\sum_{m, n=0}^{\infty} \frac{4 \lambda^{-2 n-1} \mu^{-2 m-1}}{(2 n+1)(2 m+1)} \partial_{t_{2 n+1}} \partial_{t_{2 m+1}} F=\sum_{n=0}^{\infty} \frac{2 \lambda^{-2 n-1}}{2 n+1}\left(\sum_{m=0}^{\infty} \frac{2 \mu^{-2 m-1}}{2 m+1} \partial_{t_{2 n+1}} \partial_{t_{2 m+1}} F\right) \\
=\sum_{n=0}^{\infty} \frac{2 \lambda^{-2 n-1}}{2 n+1}\left(\mu^{2 n+1}-\partial_{t_{2 n+1}} S(\mu)\right)=-\log \frac{\lambda-\mu}{\lambda+\mu}-\sum_{n=0}^{\infty} \frac{2 \lambda^{-2 n-1}}{2 n+1} \partial_{t_{2 n+1}} S(\mu) .
\end{gathered}
$$


Thus (6.1) reduces to the following system of Hamilton-Jacobi equations:

$$
\partial_{t_{2 n+1}} S(z)=\Phi_{2 n+1}(p(z))=\Phi_{2 n+1}\left(\partial_{t_{1}} S(z)\right), \quad n=0,1, \ldots
$$

2) Let us now consider (5.4). In this case, we use the Farber polynomials $\bar{\Phi}_{n}(\bar{p}), n=1,2, \ldots$ of $\bar{p}(z)$ defined by the generating functional relation

$$
\log \frac{\bar{p}(z)-w}{z}=-\sum_{n=1}^{\infty} \frac{z^{-n}}{n} \bar{\Phi}_{n}(w) .
$$

Since $\bar{p}(z)$, like $p(z)$, is an odd function of $z$, we have the generating function

$$
\log \frac{\bar{p}(z)-w}{\bar{p}(z)+w}=-\sum_{n=0}^{\infty} \frac{2 z^{-2 n-1}}{2 n+1} \bar{\Phi}_{2 n+1}(w)
$$

of the Faber polynomials with odd indices. The rest of calculations are almost the same as in the case of 1). Thus (5.4) reduces to the following Hamilton-Jacobi equations:

$$
\partial_{\bar{t}_{2 n+1}} \bar{S}(z)=\bar{\Phi}_{2 n+1}(\bar{p}(z))=\bar{\Phi}_{2 n+1}\left(\partial_{\bar{t}_{1}} \bar{S}(z)\right), \quad n=0,1, \ldots
$$

3) We can deal with (5.5) in much the same way as the case of 1). We start from the logarithmic form

$$
\log \frac{p(\lambda)-\partial_{t_{1}} \bar{S}(\mu)}{p(\lambda)+\partial_{t_{1}} \bar{S}(\mu)}=\sum_{n, m=0}^{\infty} \frac{4 \lambda^{-2 n-1} \mu^{-2 m-1}}{(2 n+1)(2 m+1)} \partial_{t_{2 n+1}} \partial_{\bar{t}_{2 m+1}} F
$$

of (5.5). By (6.3), the left hand side can be expressed as

$$
\log \frac{p(\lambda)-\partial_{t_{1}} \bar{S}(\mu)}{p(\lambda)+\partial_{t_{1}} \bar{S}(\mu)}=-\sum_{n=0}^{\infty} \frac{2 \lambda^{-2 n-1}}{2 n+1} \Phi_{2 n+1}\left(\partial_{t_{1}} \bar{S}(\mu)\right) .
$$

The right hand side can be written as

$$
\sum_{n, m=0}^{\infty} \frac{4 \lambda^{-2 n-1} \mu^{-2 m-1}}{(2 n+1)(2 m+1)} \partial_{t_{2 n+1}} \partial_{\bar{t}_{2 m+1}} F=-\sum_{n=0}^{\infty} \frac{2 \lambda^{-2 n-1}}{2 n+1} \partial_{t_{2 n+1}} \bar{S}(\mu)
$$

Thus (5.5) reduces to Hamilton-Jacobi equations of the form

$$
\partial_{t_{2 n+1}} \bar{S}(z)=\Phi_{2 n+1}\left(\partial_{t_{1}} \bar{S}(z)\right), \quad n=0,1, \ldots
$$

4) (5.6) can be treated in the same way as (5.4), and boils down to the following HamiltonJacobi equations:

$$
\partial_{\bar{t}_{2 n+1}} S(z)=\bar{\Phi}_{2 n+1}\left(\partial_{\bar{t}_{1}} S(z)\right), \quad n=0,1,2, \ldots
$$

We have thus derived the Hamilton-Jacobi equations (6.4), (6.5), (6.6) and (6.7) from the dispersionless Hirota equations (5.3), (5.4), (5.5) and (5.6). Since what we have done is simply to expand generating functions, this procedure can be reversed. The equivalence of these two systems are thus proven.

As a final remark, we note that these four sets of Hamilton-Jacobi equations can be packed into total differential equations of the form

$$
d S(z)=S^{\prime}(z) d z+\sum_{n=0}^{\infty} \Phi_{2 n+1}\left(\partial_{t_{1}} S(z)\right) d t_{2 n+1}+\sum_{n=0}^{\infty} \bar{\Phi}_{2 n+1}\left(\partial_{\bar{t}_{1}} S(z)\right) d \bar{t}_{2 n+1},
$$




$$
d \bar{S}(z)=\bar{S}^{\prime}(z) d z+\sum_{n=0}^{\infty} \Phi_{2 n+1}\left(\partial_{t_{1}} \bar{S}(z)\right) d t_{2 n+1}+\sum_{n=0}^{\infty} \bar{\Phi}_{2 n+1}\left(\partial_{\bar{t}_{1}} \bar{S}(z)\right) d \bar{t}_{2 n+1}
$$

where $S^{\prime}(z)$ and $\bar{S}^{\prime}(z)$ denote the $z$-derivative of $S(z)$ and $\bar{S}(z)$,

$$
S^{\prime}(z)=\frac{\partial S(z)}{\partial z}, \quad \bar{S}^{\prime}(z)=\frac{\partial \bar{S}(z)}{\partial z} .
$$

\section{Dispersionless Lax equations}

The Hamilton-Jacobi equations (6.4), (6.5), (6.6) and (6.7) yield a system of "dispersionless Lax equations" (i.e., Lax equations with respect to Poisson brackets rather than usual commutators) for the inverse functions $\mathcal{L}(p), \overline{\mathcal{L}}(\bar{p})$ of $p(z), \bar{p}(z)$. Since the derivation is almost the same as the case of the dispersionless KP and Toda hierarchies [10], we here show just an outline of the results, specifying some new aspects.

\subsection{How to derive dispersionless Lax equations}

Let $z=\mathcal{L}(p)$ denote the inverse function of $p=p(z)$ in a neighborhood of $z=\infty$. This is an odd function and has Laurent expansion of the form

$$
\mathcal{L}(p)=p+O\left(p^{-1}\right)
$$

in a neighborhood of $p=\infty$. We now substitute $z=\mathcal{L}(p)$ into (6.8) and note that (5.7) implies the relation

$$
\left.\partial_{\bar{t}_{1}} S(z)\right|_{z=\mathcal{L}(p)}=\left.\frac{u}{\partial_{t_{1}} S(z)}\right|_{z=\mathcal{L}(p)}=\frac{u}{p}
$$

This yields the equation

$$
d \mathcal{S}(p)=\mathcal{M}(p) d \mathcal{L}(p)+p d t_{1}+\sum_{n=1}^{\infty} \Phi_{2 n+1}(p) d t_{2 n+1}+\frac{u}{p} d \bar{t}_{1}+\sum_{n=1}^{\infty} \bar{\Phi}_{2 n+1}\left(\frac{u}{p}\right) d \bar{t}_{2 n+1},
$$

where

$$
\mathcal{S}(p)=S(\mathcal{L}(p)), \quad \mathcal{M}(p)=S^{\prime}(\mathcal{L}(p)) .
$$

Similarly, we can consider the inverse function $z=\overline{\mathcal{L}}(\bar{p})$ of $\bar{p}=\bar{p}(z)$, which is an odd function of $\bar{p}$ and has Laurent expansion of the form

$$
\overline{\mathcal{L}}(\bar{p})=\bar{p}+O\left(\bar{p}^{-1}\right)
$$

in a neighborhood of $\bar{p}=\infty$. Since (5.7) implies that

$$
\left.\partial_{t_{1}} \bar{S}(z)\right|_{z=\overline{\mathcal{L}}(\bar{p})}=\left.\frac{u}{\partial_{\bar{t}_{1}} \bar{S}(z)}\right|_{z=\overline{\mathcal{L}}(\bar{p})}=\frac{u}{\bar{p}}
$$

(6.9) turns into the equation

$$
d \overline{\mathcal{S}}(\bar{p})=\overline{\mathcal{M}}(\bar{p}) d \overline{\mathcal{L}}(\bar{p})+\frac{u}{\bar{p}} d t_{1}+\sum_{n=1}^{\infty} \Phi_{2 n+1}\left(\frac{u}{\bar{p}}\right) d t_{2 n+1}+\bar{p} d \bar{t}_{1}+\sum_{n=1}^{\infty} \bar{\Phi}_{2 n+1}(\bar{p}) d \bar{t}_{2 n+1},
$$


where

$$
\overline{\mathcal{S}}(\bar{p})=\bar{S}(\overline{\mathcal{L}}(\bar{p})), \quad \overline{\mathcal{M}}(\bar{p})=\bar{S}^{\prime}(\overline{\mathcal{L}}(\bar{p})) .
$$

$\mathcal{L}(p), \bar{L}(\bar{p})$ and $\mathcal{M}(p), \overline{\mathcal{M}}(\bar{p})$ are dispersionless analogues of the Lax and Orlov-Schulman operators of the KP and Toda hierarchies [10]. Actually, as we show below, the present situation is more complicated than the case of the KP and Toda hierarchies because of presence of two "spatial variables" $t_{1}$ and $\bar{t}_{1}$.

$p$ and $\bar{p}$ in (7.1) and (7.2) are a priori independent. To derive dispersionless Lax equations, we have to set the algebraic relation

$$
p \bar{p}=u
$$

between $p$ and $\bar{p}$. Note here that the Faber polynomials in the coefficients of (7.1) and (7.2) thereby coincide as

$$
\Phi_{2 n+1}(p)=\Phi_{2 n+1}(u / \bar{p}), \quad \bar{\Phi}_{2 n+1}(u / p)=\bar{\Phi}_{2 n+1}(\bar{p}) .
$$

Therefore we have the well-defined 1-form

$$
\Theta=p d t_{1}+\sum_{n=1}^{\infty} \Phi_{2 n+1}(p) d t_{2 n+1}+\bar{p} d \bar{t}_{1}+\sum_{n=1}^{\infty} \bar{\Phi}_{2 n+1}(\bar{p}) d \bar{t}_{2 n+1}
$$

and the equation

$$
d \mathcal{S}(p)-\mathcal{M}(p) d \mathcal{L}(p)=\Theta=d \overline{\mathcal{S}}(\bar{p})-\overline{\mathcal{M}}(\bar{p}) d \overline{\mathcal{L}}(\bar{p})
$$

of 1-forms, from which we obtain the equation

$$
d \mathcal{L}(p) \wedge d \mathcal{M}(p)=d \overline{\mathcal{L}}(\bar{p}) \wedge d \overline{\mathcal{M}}(\bar{p})
$$

of 2-forms.

Having these equations, we can now resort to the standard procedure [10] to derive dispersionless Lax equations. Actually, because of the manifest symmetry between $\boldsymbol{t}, p, \mathcal{L}(p), \mathcal{M}(p)$, $\Phi_{2 n+1}(p)$ and $\overline{\boldsymbol{t}}, \bar{p}, \overline{\mathcal{L}}(\bar{p}), \overline{\mathcal{M}}(\bar{p}), \bar{\Phi}_{2 n+1}(\bar{p})$, we can formulate the dispersionless Lax equations in two different ways. A formulation is based on the Poisson bracket

$$
\{f, g\}_{p, t_{1}}=\frac{\partial f}{\partial p} \frac{\partial g}{\partial t_{1}}-\frac{\partial f}{\partial t_{1}} \frac{\partial g}{\partial p}
$$

for the canonical pair $\left(p, t_{1}\right)$. We have the dispersionless Lax equations

$$
\partial_{t_{2 n+1}} \mathcal{K}(p)=\left\{\Phi_{2 n+1}(p), \mathcal{K}(p)\right\}_{p, t_{1}}, \quad \partial_{\bar{t}_{2 n+1}} \mathcal{K}(p)=\left\{\bar{\Phi}_{2 n+1}(u / p), \mathcal{K}(p)\right\}_{p, t_{1}}
$$

for $\mathcal{K}(p)=\mathcal{L}(p), \mathcal{M}(p), \overline{\mathcal{L}}(u / p), \overline{\mathcal{M}}(u / p)$ and the canonical commutation relations

$$
\{\mathcal{L}(p), \mathcal{M}(p)\}_{p, t_{1}}=1, \quad\{\overline{\mathcal{L}}(u / p), \overline{\mathcal{M}}(u / p)\}_{p, t_{1}}=1 .
$$

Another formulation is based on the Poisson bracket

$$
\{f, g\}_{\bar{p}, \bar{t}_{1}}=\frac{\partial f}{\partial \bar{p}} \frac{\partial g}{\partial \bar{t}_{1}}-\frac{\partial f}{\partial \bar{t}_{1}} \frac{\partial g}{\partial \bar{p}} .
$$

for the canonical pair $\left(\bar{p}, \bar{t}_{1}\right)$. We have the dispersionless Lax equations

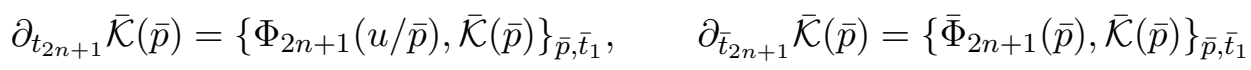

for $\overline{\mathcal{K}}(\bar{p})=\overline{\mathcal{L}}(\bar{p}), \overline{\mathcal{M}}(\bar{p}), \mathcal{L}(u / \bar{p}), \mathcal{M}(u / \bar{p})$ and the canonical commutation relations

$$
\{\overline{\mathcal{L}}(\bar{p}), \overline{\mathcal{M}}(\bar{p})\}_{\bar{p}, \bar{t}_{1}}=1, \quad\{\mathcal{L}(u / \bar{p}), \mathcal{M}(u / \bar{p})\}_{\bar{p}, \bar{t}_{1}}=1 .
$$




\subsection{How to determine Hamiltonians}

For these dispersionless Lax equations to form a closed system, the Hamiltonians have to be written in terms of the $L$-functions.

We can read off such an expression of the Hamiltonians from the Hamilton-Jacobi equations themselves. Let us examine (6.4). Substituting $z=\mathcal{L}(p)$ into this equation yields

$$
\Phi_{2 n+1}(p)=\left.\partial_{t_{2 n+1}} S(z)\right|_{z=\mathcal{L}(p)}=\mathcal{L}(p)^{2 n+1}+O\left(\mathcal{L}(p)^{-1}\right),
$$

which implies that $\Phi_{2 n+1}(p)$ coincides with the "polynomial part" of $\mathcal{L}(p)^{2 n+1}$ :

$$
\Phi_{2 n+1}(p)=\left(\mathcal{L}(p)^{2 n+1}\right)_{\geq 0} .
$$

In the same way, (6.7) yields

$$
\bar{\Phi}_{2 n+1}(\bar{p})=\left.\partial_{\bar{t}_{2 n+1}} \bar{S}(z)\right|_{z=\overline{\mathcal{L}}(\bar{p})}=\overline{\mathcal{L}}(\bar{p})^{2 n+1}+O\left(\bar{p}^{-1}\right),
$$

so that $\bar{\Phi}_{2 n+1}(\bar{p})$ is given by the polynomial part of the powers of $\overline{\mathcal{L}}(\bar{p})$ :

$$
\bar{\Phi}_{2 n+1}(\bar{p})=\left(\overline{\mathcal{L}}(\bar{p})^{2 n+1}\right)_{\geq 0} .
$$

Actually, (7.5) and (7.6) can be derived from the definition of the Faber polynomials as well. As regards $\Phi_{2 n+1}(p)$, the defining relation (6.2) differentiated by $z$ yields the identity

$$
\frac{p^{\prime}(z)}{p(z)-w}=\sum_{n=1}^{\infty} z^{-n-1} \Phi_{n}(w) .
$$

Therefore, by Cauchy's integral theorem, we have

$$
\Phi_{n}(w)=\oint_{C} \frac{d z}{2 \pi i} \frac{z^{n} p^{\prime}(z)}{p(z)-w}=\oint_{|p|=R} \frac{d p}{2 \pi i} \frac{\mathcal{L}(p)^{n}}{p-w},
$$

where $C$ is the image of the circle $|p|=R$ by the map $z=\mathcal{L}(p)$, being a simple curve for a sufficiently large value of $R$, and $w$ is understood to sit on the near side, i.e., $|w|<R$. If we insert the Laurent expansion of $\mathcal{L}(p)$ in this expression, we obtain the algebraic expression (7.5). In particular, (7.5) holds for the Faber polynomials with even indices as well, though we do not use them here. Similarly, we have an integral formula of $\bar{\Phi}_{n}(\bar{p})$, which implies the algebraic expression (7.6) and its counterpart for even indices.

\section{Another approach to dispersionless limit}

In this section, we present another approach that was taken in Takasaki's previous work on the 1-BKP hierarchy [9]. This approach is based on quasi-classical (or "WKB") approximation of auxiliary linear equations (see the review [10] and references cited therein). As expected, this approach leads to the same Hamilton-Jacobi equations as derived from the differential Fay identities.

\subsection{Wave function and auxiliary linear equations}

Since we have two vertex operators $X(\boldsymbol{t}, z)$ and $X(\overline{\boldsymbol{t}}, z)$, we can define two wave functions:

$$
\Psi(\boldsymbol{t}, \overline{\boldsymbol{t}}, z)=\frac{X(\boldsymbol{t}, z) \tau(\boldsymbol{t}, \overline{\boldsymbol{t}})}{\tau(\boldsymbol{t}, \overline{\boldsymbol{t}})}, \quad \bar{\Psi}(\boldsymbol{t}, \overline{\boldsymbol{t}}, z)=\frac{X(\overline{\boldsymbol{t}}, z) \tau(\boldsymbol{t}, \overline{\boldsymbol{t}})}{\tau(\boldsymbol{t}, \overline{\boldsymbol{t}})} .
$$


More explicitly,

$$
\Psi(\boldsymbol{t}, \overline{\boldsymbol{t}}, z)=\frac{\tau\left(\boldsymbol{t}-2\left[z^{-1}\right], \overline{\boldsymbol{t}}\right)}{\tau(\boldsymbol{t}, \overline{\boldsymbol{t}})} e^{\xi(\boldsymbol{t}, z)}, \quad \bar{\Psi}(\boldsymbol{t}, \overline{\boldsymbol{t}}, z)=\frac{\tau\left(\boldsymbol{t}, \overline{\boldsymbol{t}}-2\left[z^{-1}\right]\right)}{\tau(\boldsymbol{t}, \overline{\boldsymbol{t}})} e^{\xi(\overline{\boldsymbol{t}}, z)} .
$$

The bilinear equation (2.1) for the tau function thereby turns into the bilinear equation

$$
\oint \frac{d z}{2 \pi i z} \Psi\left(\boldsymbol{t}^{\prime}, \overline{\boldsymbol{t}}^{\prime}, z\right) \Psi(\boldsymbol{t}, \overline{\boldsymbol{t}},-z)=\oint \frac{d z}{2 \pi i z} \bar{\Psi}\left(\boldsymbol{t}^{\prime}, \overline{\boldsymbol{t}}^{\prime}, z\right) \bar{\Psi}(\boldsymbol{t}, \overline{\boldsymbol{t}},-z)
$$

for the wave functions. Note that the role of "dual wave functions" in the KP and Toda hierarchies are now played by $\Psi(\boldsymbol{t}, \overline{\boldsymbol{t}},-z)$ and $\bar{\Psi}(\boldsymbol{t}, \overline{\boldsymbol{t}},-z)$. Let us mention that such a pair of wave functions for the 2-BKP hierarchy were first considered by Shiota [7] in an algebro-geometric framework.

As we shall show below, these two wave functions satisfy the following three sets of "auxiliary linear equations."

1. Linear equations of the 1 -BKP type in the $\boldsymbol{t}$-sector:

$$
\left(\partial_{t_{2 n+1}}-B_{2 n+1}\left(\partial_{t_{1}}\right)\right) \Psi=0, \quad n=0,1,2, \ldots,
$$

where $B_{2 n+1}\left(\partial_{t_{1}}\right)$ is a differential operator in $t_{1}$ of the form $\partial_{t_{1}}^{2 n+1}+\cdots$ without 0 -th order term, i.e.,

$$
B_{2 n+1}\left(\partial_{t_{1}}\right) 1=0
$$

2. Linear equations of the 1-BKP type in the $\overline{\boldsymbol{t}}$-sector:

$$
\left(\partial_{\bar{t}_{2 n+1}}-\bar{B}_{2 n+1}\left(\partial_{\bar{t}_{1}}\right)\right) \Psi=0, \quad n=0,1,2, \ldots,
$$

where $\bar{B}_{2 n+1}\left(\partial_{\bar{t}_{1}}\right)$ is a differential operator in $t_{1}$ of the form $\partial_{\bar{t}_{1}}^{2 n+1}+\cdots$ without 0 -th order term, i.e.,

$$
\bar{B}_{2 n+1}\left(\partial_{\bar{t}_{1}}\right) 1=0 .
$$

3. Two-dimensional Schrödinger equation of the Novikov-Veselov type [24]:

$$
\left(\partial_{t_{1}} \partial_{\bar{t}_{1}}-u\right)=0
$$

where $u=-2 \partial_{t_{1}} \partial_{\bar{t}_{1}} \log \tau$.

Note that the condition on the 0 -th order term of $B_{2 n+1}\left(\partial_{t_{1}}\right)$ and $\bar{B}_{2 n+1}\left(\partial_{\bar{t}_{1}}\right)$ is the same as the condition that characterizes the $B$-operators of the 1-BKP hierarchy $[4,6]$. This is another way to see that the 2-BKP hierarchy contains two copies of the 1-BKP hierarchy.

\subsection{How to derive auxiliary linear equations}

These auxiliary linear equations are derived from the bilinear equation (8.1) by the standard method $[6,22,29,30]$ based on the following

Lemma 1. If $R(\boldsymbol{t}, \overline{\boldsymbol{t}}, z)$ and $\bar{R}(\boldsymbol{t}, \overline{\boldsymbol{t}}, z)$ are a pair of Laurent series of $z$ of the form

$$
R(\boldsymbol{t}, \overline{\boldsymbol{t}}, z)=O\left(z^{-1}\right) e^{\xi(\boldsymbol{t}, z)}, \quad \bar{R}(\boldsymbol{t}, \overline{\boldsymbol{t}}, z)=O\left(z^{-1}\right) e^{\xi(\overline{\boldsymbol{t}}, z)},
$$

and satisfy the bilinear equation

$$
\oint \frac{d z}{2 \pi i z} R\left(\boldsymbol{t}^{\prime}, \overline{\boldsymbol{t}}^{\prime}, z\right) \Psi(\boldsymbol{t}, \overline{\boldsymbol{t}},-z)=\oint \frac{d z}{2 \pi i z} \bar{R}\left(\boldsymbol{t}^{\prime}, \overline{\boldsymbol{t}}^{\prime}, z\right) \bar{\Psi}(\boldsymbol{t}, \overline{\boldsymbol{t}},-z),
$$

then $R(\boldsymbol{t}, \overline{\boldsymbol{t}}, z)=\bar{R}(\boldsymbol{t}, \overline{\boldsymbol{t}}, z)=0$. 
We refer the proof of this lemma to the references $[6,22,29,30]$ and illustrate usage of this lemma for the case of (8.2). Differentiating (8.1) by $t_{2 n+1}$ yields

$$
\oint \frac{d z}{2 \pi i z} \partial_{t_{2 n+1}^{\prime}} \Psi\left(\boldsymbol{t}^{\prime}, \overline{\boldsymbol{t}}^{\prime}, z\right) \Psi(\boldsymbol{t}, \overline{\boldsymbol{t}},-z)=\oint \frac{d z}{2 \pi i z} \partial_{t_{2 n+1}^{\prime}} \bar{\Psi}\left(\boldsymbol{t}^{\prime}, \overline{\boldsymbol{t}}^{\prime}, z\right) \bar{\Psi}(\boldsymbol{t}, \overline{\boldsymbol{t}},-z) .
$$

Similar bilinear equations hold for higher derivatives as well. In particular, we have

$$
\oint \frac{d z}{2 \pi i z} B\left(\partial_{t_{1}}\right) \Psi\left(\boldsymbol{t}^{\prime}, \overline{\boldsymbol{t}}^{\prime}, z\right) \Psi(\boldsymbol{t}, \overline{\boldsymbol{t}},-z)=\oint \frac{d z}{2 \pi i z} B\left(\partial_{t_{1}}\right) \bar{\Psi}\left(\boldsymbol{t}^{\prime}, \overline{\boldsymbol{t}}^{\prime}, z\right) \bar{\Psi}(\boldsymbol{t}, \overline{\boldsymbol{t}},-z)
$$

for any choice of differential operator $B\left(\partial_{t_{1}}\right)$ in $t_{1}$. This implies that the bilinear equation in the lemma is satisfied by

$$
\begin{aligned}
R(\boldsymbol{t}, \overline{\boldsymbol{t}}, z) & =\partial_{t_{2 n+1}} \Psi(\boldsymbol{t}, \overline{\boldsymbol{t}}, z)-B\left(\partial_{t_{1}}\right) \Psi(\boldsymbol{t}, \overline{\boldsymbol{t}}, z), \\
\bar{R}(\boldsymbol{t}, \overline{\boldsymbol{t}}, z) & =\partial_{t_{2 n+1}} \bar{\Psi}(\boldsymbol{t}, \overline{\boldsymbol{t}}, z)-B\left(\partial_{t_{1}}\right) \bar{\Psi}(\boldsymbol{t}, \overline{\boldsymbol{t}}, z) .
\end{aligned}
$$

If $B\left(\partial_{t_{1}}\right)$ is chosen to be an operator $B_{2 n+1}\left(\partial_{t_{1}}\right)$ for which other conditions of the lemma are satisfied, then $R(\boldsymbol{t}, \overline{\boldsymbol{t}}, z)$ and $\bar{R}(\boldsymbol{t}, \overline{\boldsymbol{t}}, z)$ automatically vanish, thus we obtain (8.2). The extra condition on the 0 -th order term of $B_{2 n+1}\left(\partial_{t_{1}}\right)$ turns out to be satisfied because (8.1) reduces to the bilinear equation of the 1-BKP hierarchy in the $\boldsymbol{t}$-sector upon setting $\overline{\boldsymbol{t}}^{\prime}=\overline{\boldsymbol{t}}$.

(8.3) and (8.4) can be derived in the same way.

\subsection{Lax and zero-curvature equations}

We now consider a "scalar" Lax formalism of the 2-BKP hierarchy on the basis of the auxiliary linear equations presented above. This Lax formalism is conceptually parallel to the algebrogeometric framework based on "two-point" (or "two-puncture") Baker-Akhiezer functions [7, 8]. In spite of some unusual aspects as we see below, such a scalar Lax formalism is more suited to the consideration of dispersionless limit than the "matrix" Lax formalism of Kac and van de Leur [22].

Let us first consider zero-curvature equations for the $B$-operators. As pointed out by Krichever [8], the $B$-operators satisfy the modified "zero-curvature" equations

$$
\left[\partial_{t_{2 m+1}}-B_{2 m+1}\left(\partial_{t_{1}}\right), \partial_{\bar{t}_{2 n+1}}-\bar{B}_{2 n+1}\left(\partial_{\bar{t}_{1}}\right)\right]=D_{m n}\left(\partial_{t}, \partial_{\bar{t}_{1}}\right)\left(\partial_{t_{1}} \partial_{\bar{t}_{1}}-u\right)
$$

where $D_{m n}\left(\partial_{t_{1}}, \partial_{\bar{t}_{1}}\right)$ are differential operators in both $t_{1}$ and $\bar{t}_{1}$, alongside the usual zerocurvature equations

$$
\begin{aligned}
& {\left[\partial_{t_{2 m+1}}-B_{2 m+1}\left(\partial_{t_{1}}\right), \partial_{t_{2 n+1}}-B_{2 n+1}\left(\partial_{t_{1}}\right)\right]=0} \\
& {\left[\partial_{\bar{t}_{2 m+1}}-\bar{B}_{2 m+1}\left(\partial_{\bar{t}_{1}}\right), \partial_{\bar{t}_{2 n+1}}-\bar{B}_{2 n+1}\left(\partial_{\bar{t}_{1}}\right)\right]=0}
\end{aligned}
$$

of the 1-BKP hierarchy in the $\boldsymbol{t}$ - and $\overline{\boldsymbol{t}}$-sectors. In other words, $\partial_{t_{2 m+1}}-B_{2 m+1}\left(\partial_{t_{1}}\right)$ and $\partial_{\bar{t}_{2 n+1}}-$ $\bar{B}_{2 n+1}\left(\partial_{\bar{t}_{1}}\right)$ commute "modulo" the two-dimensional Schrödinger operator $\partial_{t_{1}} \partial_{\bar{t}_{1}}-u$. We refer the details to Krichever's paper [8].

Let us turn to Lax equations. We have two $L$-operators

$$
L\left(\partial_{t_{1}}\right)=W\left(\partial_{t_{1}}\right) \partial_{t_{1}} W\left(\partial_{t_{1}}\right)^{-1}, \quad \bar{L}\left(\partial_{\bar{t}_{1}}\right)=\bar{W}\left(\partial_{\bar{t}_{1}}\right) \partial_{\bar{t}_{1}} \bar{W}\left(\partial_{\bar{t}_{1}}\right)^{-1}
$$

where $W\left(\partial_{t_{1}}\right)$ and $\bar{W}\left(\partial_{\bar{t}_{1}}\right)$ are dressing operators of the form

$$
W\left(\partial_{t_{1}}\right)=1+\sum_{j=1}^{\infty} w_{j}(\boldsymbol{t}, \overline{\boldsymbol{t}}) \partial_{t_{1}}^{-j}, \quad \bar{W}\left(\partial_{\bar{t}_{1}}\right)=1+\sum_{j=1}^{\infty} \bar{w}_{j}(\boldsymbol{t}, \overline{\boldsymbol{t}}) \partial_{\bar{t}_{1}}^{-j}
$$


that are related to the wave functions as

$$
\Psi(\boldsymbol{t}, \overline{\boldsymbol{t}}, z)=W\left(\partial_{t_{1}}\right) e^{\xi(\boldsymbol{t}, z)}, \quad \bar{\Psi}(\boldsymbol{t}, \overline{\boldsymbol{t}}, z)=\bar{W}\left(\partial_{\bar{t}_{1}}\right) e^{\xi(\overline{\boldsymbol{t}}, z)}
$$

Substituting this expression of the wave functions into (8.2) and (8.3) yields the so called Sato equations

$$
\begin{aligned}
\frac{\partial W\left(\partial_{t_{1}}\right)}{\partial t_{2 n+1}} & =B_{2 n+1}\left(\partial_{t_{1}}\right) W\left(\partial_{t_{1}}\right)-W\left(\partial_{t_{1}}\right) \partial_{t_{1}}^{2 n+1}, \\
\frac{\partial \bar{W}\left(\partial_{\bar{t}_{1}}\right)}{\partial \bar{t}_{2 n+1}} & =\bar{B}_{2 n+1}\left(\partial_{\bar{t}_{1}}\right) \bar{W}\left(\partial_{\bar{t}_{1}}\right)-\bar{W}\left(\partial_{\bar{t}_{1}}\right) \partial_{\bar{t}_{1}}^{2 n+1},
\end{aligned}
$$

which in turn imply the following expression of the $B$-operators:

$$
B_{2 n+1}\left(\partial_{t_{1}}\right)=\left(L\left(\partial_{t_{1}}\right)^{2 n+1}\right)_{\geq 0}, \quad \bar{B}_{2 n+1}\left(\partial_{\bar{t}_{1}}\right)=\left(\bar{L}\left(\partial_{\bar{t}_{1}}\right)^{2 n+1}\right)_{\geq 0},
$$

where ()$_{\geq 0}$ denotes the differential operator part of a pseudo-differential operator. Since $\Psi(\boldsymbol{t}, \overline{\boldsymbol{t}}, z)$ and $\bar{\Psi}(\boldsymbol{t}, \overline{\boldsymbol{t}}, z)$ satisfy the linear equations

$$
L\left(\partial_{t_{1}}\right) \Psi(\boldsymbol{t}, \overline{\boldsymbol{t}}, z)=z \Psi(\boldsymbol{t}, \overline{\boldsymbol{t}}, z), \quad \bar{L}(\boldsymbol{t}, \overline{\boldsymbol{t}}, z) \bar{\Psi}(\boldsymbol{t}, \overline{\boldsymbol{t}}, z)=z \bar{\Psi}(\boldsymbol{t}, \overline{\boldsymbol{t}}, z)
$$

alongside (8.2) and (8.3), we have the Lax equations

$$
\frac{\partial L\left(\partial_{t_{1}}\right)}{\partial t_{2 n+1}}=\left[B_{2 n+1}\left(\partial_{t_{1}}\right), L\left(\partial_{t_{1}}\right)\right], \quad \frac{\partial \bar{L}\left(\partial_{\bar{t}_{1}}\right)}{\partial \bar{t}_{2 n+1}}=\left[\bar{B}_{2 n+1}\left(\partial_{\bar{t}_{1}}\right), \bar{L}\left(\partial_{\bar{t}_{1}}\right)\right]
$$

which give the standard Lax representation of the 1-BKP hierarchy in the $\boldsymbol{t}$ - and $\overline{\boldsymbol{t}}$-sector, respectively.

It is, however, not so straightforward to derive Lax equations of the form

$$
\frac{\partial L\left(\partial_{t_{1}}\right)}{\partial \bar{t}_{2 n+1}}=\left[*, L\left(\partial_{t_{1}}\right)\right], \quad \frac{\partial \bar{L}\left(\partial_{\bar{t}_{1}}\right)}{\partial t_{2 n+1}}=\left[*, \bar{L}\left(\partial_{\bar{t}_{1}}\right)\right]
$$

Those Lax equations can be obtained from auxiliary linear equations of the form

$$
\left(\bar{Q}_{2 n+1}\left(\partial_{t_{1}}\right) \partial_{\bar{t}_{2 n+1}}-\bar{P}_{2 n+1}\left(\partial_{t_{1}}\right)\right) \Psi=0, \quad\left(Q_{2 n+1}\left(\partial_{\bar{t}_{1}}\right) \partial_{t_{2 n+1}}-P_{2 n+1}\left(\partial_{\bar{t}_{1}}\right)\right) \Psi=0
$$

where $\bar{P}_{2 n+1}\left(\partial_{t_{1}}\right), \bar{Q}_{2 n+1}\left(\partial_{t_{1}}\right)$ and $P_{2 n+1}\left(\partial_{\bar{t}_{1}}\right), Q_{2 n+1}\left(\bar{t}_{1}\right)$ are differential operators in $t_{1}$ and $\bar{t}_{1}$, respectively. One can derive these linear equations by the same reasoning as in the case of (8.4). Note that (8.4) amounts to the case of $n=1$ with

$$
\bar{Q}_{1}\left(\partial_{t_{1}}\right)=\partial_{t_{1}}, \quad \bar{P}_{1}\left(\partial_{t_{1}}\right)=u, \quad Q_{1}\left(\partial_{\bar{t}_{1}}\right)=\partial_{\bar{t}_{1}}, \quad P_{1}\left(\partial_{\bar{t}_{1}}\right)=u
$$

Since (8.6) and (8.8) are compatible, the $L$-operators turn out to satisfy the Lax equations

$$
\begin{aligned}
& \frac{\partial L\left(\partial_{t_{1}}\right)}{\partial \bar{t}_{2 n+1}}=\left[\bar{Q}_{2 n+1}\left(\partial_{t_{1}}\right)^{-1} \bar{P}_{2 n+1}\left(\partial_{t_{1}}\right), L\left(\partial_{t_{1}}\right)\right], \\
& \frac{\partial \bar{L}\left(\partial_{\bar{t}_{1}}\right)}{\partial t_{2 n+1}}=\left[Q_{2 n+1}\left(\partial_{\bar{t}_{1}}\right)^{-1} P_{2 n+1}\left(\partial_{\bar{t}_{1}}\right), \bar{L}\left(\partial_{\bar{t}_{1}}\right)\right] .
\end{aligned}
$$




\subsection{Quasi-classical approximation of wave functions}

We now consider the limit as $\hbar \rightarrow 0$. If the rescaled $\tau$-function $\tau_{\hbar}(\boldsymbol{t}, \overline{\boldsymbol{t}})$ behaves like $(5.1)$, the associated wave functions

$$
\Psi_{\hbar}(\boldsymbol{t}, \overline{\boldsymbol{t}}, z)=\frac{\tau_{\hbar}\left(\boldsymbol{t}-2 \hbar\left[z^{-1}\right], \overline{\boldsymbol{t}}\right)}{\tau_{\hbar}(\boldsymbol{t}, \overline{\boldsymbol{t}})} e^{\hbar^{-1} \xi(\boldsymbol{t}, z)}, \quad \bar{\Psi}_{\hbar}(\boldsymbol{t}, \overline{\boldsymbol{t}}, z)=\frac{\tau_{\hbar}\left(\boldsymbol{t}, \overline{\boldsymbol{t}}-2 \hbar\left[z^{-1}\right]\right)}{\tau_{\hbar}(\boldsymbol{t}, \overline{\boldsymbol{t}})} e^{\hbar^{-1} \xi(\overline{\boldsymbol{t}}, z)}
$$

turn out to take the quasi-classical form

$$
\Psi_{\hbar}(\boldsymbol{t}, \overline{\boldsymbol{t}}, z)=\exp \left(\hbar^{-1} S(\boldsymbol{t}, \overline{\boldsymbol{t}}, z)+O(1)\right), \quad \bar{\Psi}_{\hbar}(\boldsymbol{t}, \overline{\boldsymbol{t}}, z)=\exp \left(\hbar^{-1} \bar{S}(\boldsymbol{t}, \overline{\boldsymbol{t}}, z)+O(1)\right) .
$$

To simplify notations, let us write the phase functions $S(\boldsymbol{t}, \overline{\boldsymbol{t}}, z), \bar{S}(\boldsymbol{t}, \overline{\boldsymbol{t}}, z)$ as $S(z), \bar{S}(z)$. As expected, they have the Laurent expansion

$$
\begin{aligned}
& S(z)=\sum_{n=0}^{\infty} t_{2 n+1} z^{2 n+1}-\sum_{n=0}^{\infty} \frac{2 z^{-2 n-1}}{2 n+1} \partial_{t_{2 n+1}} F, \\
& \bar{S}(z)=\sum_{n=0}^{\infty} \bar{t}_{2 n+1} z^{2 n+1}-\sum_{n=0}^{\infty} \frac{2 z^{-2 n-1}}{2 n+1} \partial_{\bar{t}_{2 n+1}} F
\end{aligned}
$$

in a neighborhood of $z=\infty$. As the time variables are rescaled, differential operators in the auxiliary linear equations are also rescaled as

$$
\partial_{t_{2 n+1}} \rightarrow \hbar \partial_{t_{2 n+1}}, \quad \partial_{\bar{t}_{2 n+1}} \rightarrow \hbar \partial_{\bar{t}_{2 n+1}}
$$

Substituting the quasi-classical form (8.10) of the wave functions into those linear equations, we obtain a set of Hamilton-Jacobi equations for the phase functions.

The fundamental auxiliary linear equations (8.2), (8.3) and (8.4) thus yield the HamiltonJacobi equations

$$
\begin{aligned}
& \partial_{t_{2 n+1}} S(z)=\mathcal{B}_{2 n+1}\left(\partial_{t_{1}} S(z)\right), \\
& \partial_{\bar{t}_{2 n+1}} S(z)=\overline{\mathcal{B}}_{2 n+1}\left(\partial_{\bar{t}_{1}} S(z)\right), \\
& \partial_{t_{1}} S(z) \cdot \partial_{\bar{t}_{1}} S(z)=u
\end{aligned}
$$

for $S(z)$ and

$$
\begin{aligned}
& \partial_{t_{2 n+1}} \bar{S}(z)=\mathcal{B}_{2 n+1}\left(\partial_{t_{1}} \bar{S}(z)\right), \\
& \partial_{\bar{t}_{2 n+1}} \bar{S}(z)=\overline{\mathcal{B}}_{2 n+1}\left(\partial_{\bar{t}_{1}} \bar{S}(z)\right), \\
& \partial_{t_{1}} \bar{S}(z) \cdot \partial_{\bar{t}_{1}} \bar{S}(z)=u
\end{aligned}
$$

for $\bar{S}(z)$. $\mathcal{B}(p)$ and $\overline{\mathcal{B}}(\bar{p})$ denote the classical limit

$$
\mathcal{B}_{2 n+1}(p)=\lim _{\hbar \rightarrow 0} B_{2 n+1}(p), \quad \overline{\mathcal{B}}_{2 n+1}(\bar{p})=\lim _{\hbar \rightarrow 0} \bar{B}_{2 n+1}(\bar{p})
$$

of the "symbols" of the corresponding differential operators.

Similarly, (8.6) yields the equations

$$
\mathcal{L}\left(\partial_{t_{1}} S(z)\right)=z, \quad \overline{\mathcal{L}}\left(\partial_{\bar{t}_{1}} \bar{S}(z)\right)=z
$$

where $\mathcal{L}(p)$ and $\overline{\mathcal{L}}(\bar{p})$, like $\mathcal{B}_{2 n+1}(p)$ and $\overline{\mathcal{B}}_{2 n+1}(\bar{p})$, are given by

$$
\mathcal{L}(p)=\lim _{\hbar \rightarrow 0} L(p), \quad \overline{\mathcal{L}}(\bar{p})=\lim _{\hbar \rightarrow 0} \bar{L}(\bar{p}) .
$$


Thus $p(z)=\partial_{t_{1}} S(z)$ and $\bar{p}(z)=\partial \bar{t}_{1} \bar{S}(z)$ turn out to be the inverse functions of $z=\mathcal{L}(p)$ and $z=\overline{\mathcal{L}}(\bar{p})$, respectively. The relation (8.5) connecting the $B$-operators and the $L$-operators turns into the relation

$$
\mathcal{B}_{2 n+1}(p)=\left(\mathcal{L}(p)^{2 n+1}\right)_{\geq 0}, \quad \overline{\mathcal{B}}_{2 n+1}(\bar{p})=\left(\overline{\mathcal{L}}(\bar{p})^{2 n+1}\right)_{\geq 0}
$$

among the corresponding phase space functions. $(\cdot)_{\geq 0}$ now stand for the polynomial part of Laurent series (of $p$ and $\bar{p}$, respectively). This, in particular, implies that $\mathcal{B}_{2 n+1}(p)$ coincide with the Farber polynomials of the inverse function $p=p(z)$ of $z=\mathcal{L}(p)$. A similar statement holds for $\overline{\mathcal{B}}_{2 n+1}(\bar{p})$ and the inverse function $\bar{p}=\bar{p}(z)$ of $z=\overline{\mathcal{L}}(\bar{p})$. Thus we have

$$
\mathcal{B}_{2 n+1}(p)=\Phi_{2 n+1}(p), \quad \overline{\mathcal{B}}_{2 n+1}(\bar{p})=\bar{\Phi}_{2 n+1}(\bar{p}) .
$$

The somewhat strange linear equations (8.8), too, turn out to have a natural interpretation. The associated Hamilton-Jacobi equations read

$$
\begin{aligned}
& \overline{\mathcal{Q}}_{2 n+1}\left(\partial_{t_{1}} S(z)\right) \partial_{\bar{t}_{2 n+1}} S(z)=\overline{\mathcal{P}}_{2 n+1}\left(\partial_{t_{1}} S(z)\right), \\
& \mathcal{Q}_{2 n+1}\left(\partial_{\bar{t}_{1}} S(z)\right) \partial_{t_{2 n+1}} S(z)=\mathcal{P}_{2 n+1}\left(\partial_{\bar{t}_{1}} S(z)\right),
\end{aligned}
$$

for $S(z)$ and

$$
\begin{aligned}
& \overline{\mathcal{Q}}_{2 n+1}\left(\partial_{t_{1}} \bar{S}(z)\right) \partial_{\bar{t}_{2 n+1}} \bar{S}(z)=\overline{\mathcal{P}}_{2 n+1}\left(\partial_{t_{1}} \bar{S}(z)\right), \\
& \mathcal{Q}_{2 n+1}\left(\partial_{\bar{t}_{1}} \bar{S}(z)\right) \partial_{t_{2 n+1}} \bar{S}(z)=\mathcal{P}_{2 n+1}\left(\partial_{\bar{t}_{1}} S(z)\right),
\end{aligned}
$$

$\bar{S}(z)$, where

$$
\begin{array}{ll}
\overline{\mathcal{Q}}_{2 n+1}(p)=\lim _{\hbar \rightarrow 0} \bar{Q}_{2 n+1}(p), & \overline{\mathcal{P}}_{2 n+1}(p)=\lim _{\hbar \rightarrow 0} \bar{P}_{2 n+1}(p), \\
\mathcal{Q}_{2 n+1}(\bar{p})=\lim _{\hbar \rightarrow 0} Q_{2 n+1}(\bar{p}), & \mathcal{P}_{2 n+1}(\bar{p})=\lim _{\hbar \rightarrow 0} P_{2 n+1}(\bar{p}) .
\end{array}
$$

For the equations of the $\overline{\boldsymbol{t}}$-flows to be consistent with the previous Hamilton-Jacobi equations, $\mathcal{P}_{2 n+1}(p), \mathcal{Q}_{2 n+1}(p)$ and $\overline{\mathcal{B}}_{2 n+1}(\bar{p})$ have to satisfy the equations

$$
\begin{aligned}
& \overline{\mathcal{Q}}_{2 n+1}\left(\partial_{t_{1}} S(z)\right)^{-1} \overline{\mathcal{P}}_{2 n+1}\left(\partial_{t_{1}} S(z)\right)=\overline{\mathcal{B}}_{2 n+1}\left(\partial_{\bar{t}_{1}} S(z)\right), \\
& \overline{\mathcal{Q}}_{2 n+1}\left(\partial_{t_{1}} \bar{S}(z)\right)^{-1} \overline{\mathcal{P}}_{2 n+1}\left(\partial_{t_{1}} \bar{S}(z)\right)=\overline{\mathcal{B}}_{2 n+1}\left(\partial_{\bar{t}_{1}} \bar{S}(z)\right) .
\end{aligned}
$$

Since $\partial_{t_{1}} S(z)$ and $\partial_{\bar{t}_{1}} S(z)$ obey the algebraic relation (8.11), these two equations reduce to a single equation of the form

$$
\overline{\mathcal{B}}_{2 n+1}(u / p)=\overline{\mathcal{Q}}_{2 n+1}(p)^{-1} \overline{\mathcal{P}}_{2 n+1}(p) .
$$

Similarly, we have the relation

$$
\mathcal{B}_{2 n+1}(u / \bar{p})=\mathcal{Q}_{2 n+1}(\bar{p})^{-1} \mathcal{P}_{2 n+1}(\bar{p}) .
$$

Actually, this is a result to be expected from (8.8), because (8.8) can be (formally) rewritten as

$$
\begin{aligned}
& \left(\partial_{\bar{t}_{2 n+1}}-\bar{Q}_{2 n+1}\left(\partial_{t_{1}}\right)^{-1} \bar{P}_{2 n+1}\left(\partial_{t_{1}}\right)\right) \Psi=0, \\
& \left(\partial_{t_{2 n+1}}-Q_{2 n+1}\left(\partial_{\bar{t}_{1}}\right)^{-1} P_{2 n+1}\left(\partial_{\bar{t}_{1}}\right)\right) \Psi=0,
\end{aligned}
$$

and for these equations to be consistent with (8.2) and (8.3), we should have

$$
\begin{aligned}
& \bar{B}_{2 n+1}\left(\partial_{\bar{t}_{1}}\right) \Psi=\bar{Q}_{2 n+1}\left(\partial_{t_{1}}\right)^{-1} \bar{P}_{2 n+1}\left(\partial_{t_{1}}\right) \Psi, \\
& B_{2 n+1}\left(\partial_{t_{1}}\right) \Psi=Q_{2 n+1}\left(\partial_{\bar{t}_{1}}\right)^{-1} P_{2 n+1}\left(\partial_{\bar{t}_{1}}\right) \Psi,
\end{aligned}
$$

which may be thought of as "quantization" of (8.12) and (8.13). 


\subsection{Dispersionless Lax equations}

In the limit as $\hbar \rightarrow 0,(8.7)$ and (8.9) turn into the dispersionless Lax equations

$$
\partial_{t_{2 n+1}} \mathcal{L}(p)=\left\{\mathcal{B}_{2 n+1}(p), \mathcal{L}(p)\right\}_{p, t_{1}}, \quad \partial_{\bar{t}_{2 n+1}} \overline{\mathcal{L}}(\bar{p})=\left\{\overline{\mathcal{B}}_{2 n+1}(\bar{p}), \overline{\mathcal{L}}(\bar{p})\right\}_{\bar{p}, \bar{t}_{1}}
$$

and

$$
\begin{aligned}
& \partial_{\bar{t}_{2 n+1}} \mathcal{L}(p)=\left\{\overline{\mathcal{Q}}_{2 n+1}(p)^{-1} \bar{P}_{2 n+1}(p), \mathcal{L}(p)\right\}_{p, t_{1}}, \\
& \partial_{t_{2 n+1}} \overline{\mathcal{L}}(\bar{p})=\left\{\mathcal{Q}_{2 n+1}(\bar{p})^{-1} \mathcal{P}_{2 n+1}(\bar{p}), \overline{\mathcal{L}}(\bar{p})\right\}_{\bar{p}, \bar{t}_{1}},
\end{aligned}
$$

respectively. In view of $(8.12)$ and $(8.13)$, we can rewrite the second two as

$$
\partial_{\bar{t}_{2 n+1}} \mathcal{L}(p)=\left\{\overline{\mathcal{B}}_{2 n+1}(u / p), \mathcal{L}(p)\right\}_{p, t_{1}}, \quad \partial_{t_{2 n+1}} \overline{\mathcal{L}}(\bar{p})=\left\{\mathcal{B}_{2 n+1}(u / \bar{p}), \overline{\mathcal{L}}(\bar{p})\right\}_{\bar{p}, \bar{t}_{1}} .
$$

Obviously, these equations coincide with the corresponding equations in (7.3) and (7.4).

\section{Acknowledgements}

I would like to thank T. Ikeda, T. Shiota and T. Takebe for cooperation. I also would like to thank L. Martinez Alonso, B. Konopelchenko, M. Mañas and A. Sorin for useful discussions during the SISSA conference "Riemann-Hilbert Problems, Integrability and Asymptotics" in September, 2005. This research was partially supported by Grant-in-Aid for Scientific Research No. 16340040 from the Japan Society for the Promotion of Science.

[1] Sato M., Soliton equations as dynamical systems on an infinite dimensional Grassmannian manifold, Surikaiseki Kenkyusho Kokyuroku (RIMS, Kyoto University), 1981, V.439, 30-46.

[2] Sato M., Sato Y., Soliton equations as dynamical systems on an infinite dimensional Grassmannian manifold, in Nonlinear Partial Differential Equations in Applied Science (1982, Tokyo), Editors H. Fujita, P.D. Lax and G. Strang, North-Holland Math. Stud., Vol. 81, Amsterdam, North-Holland, 1983, 259-271.

[3] Date E., Kashiwara M., Miwa T., Transformation groups for soliton equations. II. Vertex operators and $\tau$ functions, Proc. Japan Acad. Ser. A Math. Sci. 1981, V.57, 387-392.

[4] Date E., Jimbo M., Kashiwara M., Miwa T., Transformation groups for soliton equations. IV. A new hierarchy of soliton equations of KP-type, Phys. D, 1981/82, V.4, 343-365.

[5] Date E., Jimbo M., Kashiwara M., Miwa T., Transformation groups for soliton equations. V. Quasiperiodic solutions of the orthogonal KP equation, Publ. Res. Inst. Math. Sci., 1982, V.18, 1111-1119.

[6] Date E., Jimbo M., Kashiwara M., Miwa T., Transformation groups for soliton equations. VI. KP hierarchies of orthogonal and symplectic type, J. Phys. Soc. Japan, 1981, V.50, 3813-3818.

[7] Shiota T., Prym varieties and soliton equations, in Infinite Dimensional Lie Algebras and Groups, Editor V.G. Kac, Advanced Series in Math. Phys., Vol. 7, Singapore, World Scientific, 1989, 407-448.

[8] Krichever I., A characterization of Prym varieties, math.AG/0506238.

[9] Takasaki K., Quasi-classical limit of BKP hierarchy and W-infinity symmetries, Lett. Math. Phys., 1993, V.28, 177-185, hep-th/9301090.

[10] Takasaki K., Takebe T., Integrable hierarchies and dispersionless limit, Rev. Math. Phys., 1995, V.7, 743808 , hep-th/9405096.

[11] Bogdanov L.V., Konopelchenko B.G., On dispersionless BKP hierarchy and its reductions, J. Nonlinear Math. Phys., 2005, V.12, suppl. 1, 64-75, nlin.SI/0411046.

[12] Konopelchenko B.G., Martinez Alonso L., Ragnisco O., $\bar{\partial}$-approach to the dispersionless KP hierarchy, J. Phys. A: Math. Gen., 2001, V.34, 10209-10217, nlin.SI/0103023.

[13] Konopelchenko B., Martinez Alonso L., $\bar{\partial}$-equations, integrable deformations of quasi-conformal mappings and Whitham hierarchy, Phys. Lett. A, 2001, V.286, 161-166, nlin.SI/0103015.

[14] Konopelchenko B., Martinez Alonso L., Dispersionless scalar integrable hierarchies, Whitham hierarchy and the quasi-classical $\bar{\partial}$-dressing method, J. Math. Phys., 2001, V.43, 3807-3823. 
[15] Bogdanov L.V., Konopelchenko B.G., Martinez Alonso L., Semiclassical $\bar{\partial}$-method: generating equations for dispersionless integrable hierarchies, Teoret. Mat. Fiz., 2003, V.134, 46-54 (English transl.: Theoret. and Math. Phys., 2003, V.134, 39-46).

[16] Krichever I.M., The $\tau$-function of the universal Whitham hierarchy, matrix models and topological field theories, Comm. Pure. Appl. Math., 1994, V.47, 437-475, hep-th/9205110.

[17] Carroll R., Kodama Y., Solutions of the dispersionless Hirota equations, J. Phys. A: Math. Gen., 1995, V.28, 6373-6378, hep-th/9506007.

[18] Kostov I.K., Krichever I., Mineev-Weinstein M., Wiegmann P.B., Zabrodin A., $\tau$-function for analytic curve, in Random Matrices and Their Applications, Editors P. Bleher and A. Its, Math. Sci. Res. Inst. Publ., Vol. 40, Cambridge, Cambridge University Press, 2001, 285-299, hep-th/0005259.

[19] Zabrodin A., Dispersionless limit of Hirota equations in some problems of complex analysis, Teoret. Mat. Fiz., 2001, V.129, 239-257 (English transl.: Theoret. and Math. Phys., 2001, V.129, 1511-1525), math.CV/0104169.

[20] Boyarsky A., Marshakov A., Ruchayskiy O., Wiegmann P., Zabrodin A., Associativity equations in dispersionless integrable hierarchies, Phys. Lett. B, 2001, V.515, 483-492, hep-th/0105260.

[21] Teo L.-P., Analytic functions and integrable hierarchies - characterization of tau functions, Lett. Math. Phys., 2003, V.64, 75-92, hep-th/0305005.

[22] Kac V., van de Leur J., The geometry of spinors and the multicomponent BKP and DKP hierarchies, CRM Proc. Lect. Notes, Vol. 14, Providence, American Mathematical Society, 1998, 159-202.

[23] Date E., Jimbo M., Kashiwara M., Miwa T., Transformation groups for soliton equations. Euclidean Lie algebras and reduction of the KP hierarchy, Publ. Res. Inst. Math. Sci., 1982, V.18, 1077-1110.

[24] Novikov S., Veselov A., Finite-gap two-dimensional potential Schrödinger operators. Explicit formulas and evolution equations, Soviet Math. Dokl., 1984, V.30, 588-591;

Novikov S., Veselov A., Finite-gap two-dimensional Schrödinger operators. Potential operators, Soviet Math. Dokl., 1984, V.30, 705-708.

[25] Ueno K., Takasaki K., Toda lattice hierarchy, in Group Representations and Systems of Differential Equations (1982, Tokyo), Adv. Stud. Pure Math., Vol. 4, Amsterdam, North-Holland, 1984, 1-94.

[26] Konopelchenko B., Moro A., Integrable equations in nonlinear geometrical optics, Stud. Appl. Math., 2004, V.113, 325-352, nlin.SI/0403051.

[27] Jimbo M., Miwa T., Solitons and infinite dimensional Lie algebras, Publ. Res. Inst. Math. Sci., 1983, V.19, 943-1001.

[28] Takebe T., Representation theoretical meaning of the initial value problem for the Toda lattice hierarchy. I, Lett. Math. Phys., 1991, V.21, 77-84;

Takebe T., Representation theoretical meaning of the initial value problem for the Toda lattice hierarchy. II, Publ. Res. Inst. Math. Sci., 1991, V.27, 491-503.

[29] Date E., Jimbo M., Kashiwara M., Miwa T., Transformation groups for soliton equations, in Nonlinear Integrable Systems - Classical Theory and Quantum Theory, Editors M. Jimbo and T. Miwa, Singapore, World Scientific, 1983, 39-119.

[30] Dickey L., Soliton equations and Hamiltonian systems, Singapore, World Scientific, 1991. 